pag

Business School

WORKING PAPER SERIES

Working Paper

2014-348
On Path-Dependent Structured Funds: Complexity Does Not Always Pay (Asian versus Average Performance Funds)

Philippe Bertrand

Jean-luc Prigent

http://www.ipag.fr/fr/accueil/la-recherche/publications-WP.html

IPAG Business School

184, Boulevard Saint-Germain

75006 Paris

France 


\title{
On Path-Dependent Structured Funds: Complexity Does Not Always Pay (Asian versus Average Performance Funds)
}

\author{
Philippe Bertrand \\ CERGAM, Aix-Marseille University, \\ Aix-en-Provence, France. \\ and \\ KEDGE BS, Marseille, France. \\ Tel : 33 (0)4 91140743 \\ e-mail: philippe.bertrand@univ-amu.fr
}

\author{
Jean-luc Prigent \\ IPAG Business School, 184 Bd Saint-Germain, \\ 75006, Paris, France \\ and \\ THEMA, University of Cergy-Pontoise, \\ 33, Bd du Port, 95011, Cergy-Pontoise, France \\ Tel : 331342561 72; Fax: 33134256233 \\ e-mail: jean-luc.prigent@u-cergy.fr
}

This version: May 2014

\begin{abstract}
As emphasized by the U.S. Dodd-Frank Act and the European MiFID directive, financial institutions are required to "categorise their clients and assess their suitability for each type of investment product." In this framework, this paper examines several standard financial structured products whose performances are based on smoothing the return of an underlying asset and providing a guarantee at maturity. We use various criteria such as probabilities of providing merely the guarantee at maturity and performances with respect to Kappa measures. Surprisingly, our study reveals that funds based on averages of calls generally do better than Asian funds.
\end{abstract}

JEL classification: G11, G13, G21, D03.

Keywords: Structured financial products; portfolio insurance; Asian options; average of calls; Kappa measures.

\section{Introduction}

The suitability of complex financial products for retail investors is one of the main important issues in light of investor financial protection. As mentioned by Chang et al. (2013), the U.S. Dodd-Frank Act (enacted into law on July 21, 2010) requires that the adequacy of structured products to retail investors has to be checked. The European MiFID directive (initiated in 2004 and implemented in November 2007) has also clearly emphasized that financial institutions must categorize their clients and check the suitability of investment products to the risk attitude of customers. These latter ones are considered as "eligible counterparts" and correspond to either professional customers or retail customers. "The appropriateness of any investment advice or suggested financial transaction must still be verified before being given."1

\footnotetext{
${ }^{1}$ See Weber et al. (2013) for an illustration of how risk taking changed substantially during the period September 08 to June 09 and its connexion to MiFID directive.
} 
Among investment vehicles that can be proposed by financial institutions, structured products were introduced to provide investors with access to non-standard financial instruments such as complex options. Structured products provide an alternative to standard direct portfolio allocation. Typically, the fund can provide capital protection while allowing investors to benefit from market rises through more or less sophisticated derivatives written on a single security, a basket of securities or financial indices. Structured products were created to meet specific needs that cannot be satisfied by the financial assets available in the markets. The suitability of a given structured (pre-packaged) product within a portfolio depends on the investor's attitude toward risk (see Shefrin and Statman, 1993; Driessen and Maenhout, 2007). As emphasized by Das and Statman (2013), financial structured products are more popular in Europe than in the USA.

This is probably why most empirical studies on structured products focus on European markets, especially Switzerland and Germany. The usual approach consists in comparing prices in the primary or secondary market to theoretical fair values. Some authors have analyzed the pricing of such products. Among them are Chen and Kensinger (1990) and Chen and Sears (1990), who examine the pricing of convex instruments on the S\&P 500, Wasserfallen and Schenk (1996) and Burth et al. (2001) for the Swiss market, Stoimenov and Wilkens (2005), who investigate fair pricing of equity-linked structured products in the German private retail banking sector and Bertrand and Prigent (2012), who study the fair pricing of French financial structured products. Their results show that almost all types of equity-linked structured products are priced above their theoretical values. Usually, more complex products incorporate higher implicit premiums.

As of July 2012, assets under management on the French retail market for structured products ${ }^{2}$ amount to 48,6 billion euros, representing about $4 \%$ of the total assets under management. ${ }^{3}$ Most of them are of a convex payoff type and do not pay coupons during their lifetime. In the case of French structured products, the subscription period is generally a limited period after which the fund is issued with a fixed number of shares. The investor is then strongly advised to keep his shares until the maturity date of the fund. Structured products are closed to new subscriptions from the inception date, except for subscriptions made for the sole purpose of offsetting redemptions initiated by the holders of the fund.

Thus, there is no real secondary market: only the financial institution which issues the structured product can buy back shares sold by the retail investor. For example, during the months of September and October 2012, less than 10 million euros' worth of share were sold back by investors, that is about $0.02 \%$ of the assets under management in structured products. If the investor sells his shares before the expiry date, the price offered will depend on the market parameters on that day (net of redemption fees). It may be very different (lower or higher) from the amount obtained by straightforward application of the formula and is not forecastable.

$91 \%$ of the total amount of structured funds are managed with a capital guarantee clause. They involve portfolio insurance (PI), which has been used extensively by the financial management industry in equities, bonds and hedge funds. PI is particularly valuable during a financial downturn, allowing a given percentage of the initial portfolio value to be recovered at maturity. One of the standard portfolio insurance methods is Option Based Portfolio Insurance (OBPI), introduced by Leland and Rubinstein

\footnotetext{
${ }^{2}$ These are mutual funds, called "fonds à formule", which means that they are based on a given (technical) formula which defines the fund's payoff at maturity.

${ }^{3}$ Investors can have also access to structured products on Euronext through certificates, warrants and structured notes, which are not mutual funds but are listed on the stock exchange and tradable like shares.
} 
(1976). ${ }^{4}$ The portfolio is invested in a risky benchmark asset $S$ covered by a listed put written on it. The strike $K$ of the put is equal to a predetermined proportion of the initial investment. This amount corresponds to the capital that is insured at maturity whatever the value of $S$ at the terminal date $T .{ }^{5}$ Hereafter, we will only consider capital-guaranteed structured products.

About $32 \%$ of French capital-guaranteed structured products combine a guaranteed return at maturity with a bonus based on smoothing the price variations of a reference portfolio, typically a financial index, using two main methods. The first used by $14 \%$ of the guaranteed funds, is linked to an Asian call option. Such funds deliver part of the positive performance of the average of risky asset values during the management period. The second used by $18 \%$ of the guaranteed funds, is based on an average of past positive performances of the benchmark asset (at a given date, the performance is considered positive if the value of the risky asset is higher than the initial value of the underlying asset).

Both methods smooth the performance of the underlying asset over time. They reduce the consequences of a decline in value of the underlying instrument near or at maturity. Due to their averaging feature, such options can provide lower volatility than their underlying assets, which can be indices, baskets of stocks, baskets of indices or baskets of funds. In the remainder of the paper, we consider only funds for which the underlying asset is a financial index. ${ }^{6}$ As mentioned previously, the path-dependency derives from various averages of monthly or quarterly returns. The question, however, is wether these funds are more suitable than the standard OBPI, and which kind of fund is the best choice according to specific financial decision criteria.

Our results suggest that, for investors focusing only on expected return, Asian funds are preferred, since the expectation of the Asian fund return is higher than the expectation of the Average fund return. These investors are risk-neutral investors, which seems to contradict the fact that they simultaneously require capital protection. But, as soon as potential risk aversion or implicitly loss aversion are taken into account, the ranking is reversed. We also establish that the probability of providing merely the insured amount for the Average fund is much higher than the same probability for the Asian fund. Within the context of structured products, ending up merely at the insured level is a major concern for investors. In such case, investors obtain less than the riskless rate.

The paper is organized as follows. Section 2 is devoted to the modelling of both kinds of funds and to the study of their valuations. We first search for analytical expressions of their theoretical values. Where this is not possible, due, for example, to discrete-time variations, we rely on Monte Carlo experiments to compute their values. Section 3 compares the different types of funds using various criteria, such as basic properties of the cumulative distribution functions of their respective returns. We also introduce Kappa performance measures, among them the Omega and the Sortino ratios. As a by-product, we also compare both these funds with the most common structured financial contract, namely the OBPI fund. After studying the standard geometric Brownian motion (GBM) case, we also consider that the risky asset dynamics may involve jumps. For this purpose, we introduce a double exponential Lévy process, as in Kou and Wang (2004). We calibrate this latter stochastic process using both the S\&P 500 and the Dow Jones Euro Stoxx 50 Index data set. Technical details are available in the Appendix.

\footnotetext{
${ }^{4}$ See Bertrand and Prigent $(2005,2011)$ for more details about portfolio insurance methods and their comparison.

${ }^{5}$ Equivalently, a call option can be bought on $S$ with strike $K$ and cash held equal to the discounted value of $K$.

${ }^{6}$ This makes it easier to compare different funds, without loss of generality.
} 


\section{Description of the funds and their valuation}

In this section, we describe the two structured products and valuate their optional components. In order to further compare these two products, we assume the same initial investment, $V_{0}$, on both products, the same maturity, $T$, and the same guarantee at maturity, $V_{0}$. These contracts are written on the same underlying asset which initial price is denoted, $S_{0}$. Since the type of options used in both products are distinct, we must determine the number of options for each contract in order to meet the budget constraint. This number is denoted thereafter by $\alpha V_{0}$ since it is the usual way that those contracts are drawn up. The parameter $\alpha$ is determined through the valuation equation of the structured products and must be set at the issuance date. Therefore, the price of the option must be calculated at the issuance date. The usual practice is to base financial structured products on discrete-time monitoring. Typically, monthly or quarterly returns of a given risky asset are involved in the fund's formula.

\subsection{Guaranteed funds based on Asian options}

Asian options were introduced to take account of the average of the underlying asset values, unlike the European-type options linked to the standard OBPI method which only use value at maturity. It is not surprising, therefore, that they are being used with financial structured funds, particularly insured funds. A guaranteed fund based on discrete-time Asian options delivers a payoff defined as follows:

- After $T$ years of the management period, the initial portfolio value $V_{0}$ (which corresponds to the given initial investment) is guaranteed: $V_{T} \geq V_{0}$;

- The performance $S_{i}$ of the underlying index (for example, the Dow Jones Euro Stoxx 50 Index) with respect to the initial value $S_{0}$ is taken into account each $i$-th time period $(1 \leq i \leq n T)$ since inception. The usual frequencies observed are : monthly $(n=12)$, quarterly $(n=4)$, half-yearly $(n=2)$ and yearly $(n=1)$. Then, the final performance of the index is obtained by computing the arithmetic mean of the $n T$ performances, $S_{i} \cdot{ }^{7}$ The fund is based on a given percentage of the previous arithmetic mean, which is added to the guaranteed capital $V_{0}$. The latter percentage is adjusted in order to meet the budget constraint (i.e. the initial value of the fund must be equal to $\left.V_{0}\right)$. We express it as a percentage $\alpha_{a s}$ of the value $V_{0}$.

Thus, at maturity, the payoff $V_{T}$ of this fund is defined as follows:

$$
V_{T}=V_{0}+\alpha_{a s} V_{0}\left[A(T)-S_{0}\right]^{+},
$$

with $A(T)=\frac{1}{n T} \sum_{i=1}^{n T} S_{i}$ (notation: $x^{+}=\max (x, 0)$ ).

The term $\left[A(T)-S_{0}\right]^{+}$in expression (1) represents the payoff of a discrete arithmetic Asian Call on the index with a strike price equal to the initial price $S_{0}$ of the index. We denote its price by Call $^{\text {Asian }}\left(T, A(T), S_{0}, r\right)$ where $r$ corresponds to the riskless interest rate. Therefore, at time 0 , the budget constraint is given by:

$$
V_{0}=V_{0} e^{-r T}+\alpha_{a s} V_{0} \operatorname{Call}^{\text {Asian }}\left(T, A(T), S_{0}, r\right),
$$

\footnotetext{
${ }^{7}$ The dividends received from the index by the fund are not transferred to the investors.
} 
from which we deduce that the parameter $\alpha_{a s}$ must be chosen such that:

$$
\alpha_{a s}=\frac{\left(1-e^{-r T}\right)}{\operatorname{Call}^{\text {Asian }}\left(T, A(T), S_{0}, r\right)} .
$$

Note that the return of the strategy over the period $[0, T]$ is also defined by:

$$
\frac{V_{T}}{V_{0}}=1+\left(1-e^{-r T}\right) \frac{\left[A(T)-S_{0}\right]^{+}}{\operatorname{Call}^{\text {Asian }}\left(T, A(T), S_{0}, r\right)} .
$$

\subsection{Guaranteed funds based on average positive performances}

Instead of considering only the average of the index values from inception to expiry, as for the Asian options, account is taken of the average of past positive performances with respect to a given underlying asset value, usually the initial one $S_{0}$. The performances (monthly, quarterly or half-yearly) are defined by averages of calls with strike equal to the initial value $S_{0}$ of the underlying risky asset. The payoff of a guaranteed fund based on average (excess) performances is defined as follows:

- After $T$ years of the management period, the initial portfolio value $V_{0}$ (which corresponds to the given initial investment) is guaranteed: $V_{T} \geq V_{0}$,

- The computation of the final performance is performed at the maturity date, $T$, in the following way:

- Each semester $i$ (or month or quarter), the level $S_{i}$ of the index is recorded;

- If this level is smaller than the starting value $S_{0}$ of the index, the positive performance is equal to 0 ;

- At the end of $T$ years, the arithmetic mean of the $n T$ positive performances is computed;

- The final performance of the fund is equal to $\alpha_{a v} V_{0}$ of the previous average performance added to the guaranteed amount $V_{0}$.

Thus, at maturity, the payoff of this fund is defined as follows:

$$
V_{T}=V_{0}+\alpha_{a v} V_{0} \frac{1}{n T} \sum_{i=1}^{n T}\left(S_{i}-S_{0}\right)^{+} .
$$

Therefore, only the $S_{i}$ values of the underlying index which are higher than the initial $S_{0}$ value are taken into account in the formula.

We denote by $\operatorname{Call}(i, S, K, r)$ the price of each call option with maturity $i$, strike $K$, underlying $S$, and riskless interest rate $r$. We deduce that, at time 0 , the budget constraint is given by:

$$
V_{0}=V_{0} e^{-r T}+\alpha_{a v} V_{0} \frac{1}{n T} \sum_{i=1}^{n T} e^{-r(T-i)} \operatorname{Call}\left(i, S, S_{0}, r\right) .
$$

Thereafter, we denote:

$$
\operatorname{Call}^{\text {Average }}\left(T, S, S_{0}, r\right)=\frac{1}{n T} \sum_{i=1}^{n T} e^{-r(T-i)} \operatorname{Call}\left(i, S, S_{0}, r\right)
$$


Again, parameter $\alpha_{a v}$ is chosen such that the initial price $V_{0}$ solves equation (6):

$$
\alpha_{a v}=\frac{\left(1-e^{-r T}\right)}{\text { Call }^{\text {Average }}\left(T, S, S_{0}, r\right)} .
$$

The return of the Average strategy over the period $[0, T]$ is given by:

$$
\frac{V_{T}}{V_{0}}=1+\left(1-e^{-r T}\right) \frac{\frac{1}{n T} \sum_{i=1}^{n T}\left(S_{i}-S_{0}\right)^{+}}{\text {Call }{ }^{\text {Average }}\left(T, S, S_{0}, r\right)} .
$$

\subsection{Valuation of the Asian and Average funds}

As mentioned previously, we must find the parameters $\alpha_{a s}$ and $\alpha_{a v}$ that meet the budget constraint. For this purpose, we need to valuate the optional component of the structured product. First, we must describe the financial market. Despite the discrete-time features of structured products, we can consider that the price of the benchmark risky asset follows a continuous-time process. For the valuation, using complex dynamics corresponding to continuous-time monitoring and continuous-time information flows is the subject of a debate. When structured products are appraised, usually they are examined in the geometric Brownian motion case (GBM) for various parameter values, as it is considered in one part of this paper (see e.g. Stoimenov and Wilkens, 2005). Note also that El Karoui et al. (1998) show that we can get upper bounds for prices of both European and Asian options by using a Black-Scholes model with constant volatility under the assumption that this latter one is higher than the stochastic volatility. Consequently our approach which measures the effect of a constant volatility lying from $15 \%$ to as much as $40 \%$ is legitimate.

Second, as the GBM assumption may be questioned, we investigate what happens for other dynamics. It is well-known that, for portfolio insurance, one major risk is that sudden drops occur in the risky asset dynamics, which is not taken into account by main stochastic volatility models leading to diffusion processes without jumps. To model the risky asset dynamics, we have also chosen another rather standard financial modelling, namely the double exponential Lévy process. Why? Nowadays, this model is wellknown and rather popular due to its tractability. It is also rather flexible to fit data as illustrated by Kou and Wang (2004) and Ramezani and Zeng (2007). Of course, it involves a jump component. Note that the occurrence of jumps generates (non-continuous) volatility (see Relation 12 below).

For the illustrations, we use the GBM hypothesis for the simulations with respect to various parameter values. However, we introduce the double exponential Lévy process for numerical illustrations based on an empirical analysis on both the S\&P 500 and the Dow Jones Euro Stoxx 50. Indeed, for the empirical analysis, since the statistical results (based on the maximum-likelihood method) are in favor of the presence of jumps, we use the double exponential Lévy process instead of the standard GBM. ${ }^{8}$

The standard model is the well-known geometric Brownian motion where the stock index price dynamics is given by the following stochastic process:

$$
d S_{t}=S_{t}\left[\mu d t+\sigma d W_{t}\right]
$$

where $\mu$ and $\sigma$ are constant $(\sigma>0)$ and $W$ denotes a standard Brownian motion with respect to a given filtration $\left(\mathcal{F}_{t}\right)_{t}$.

\footnotetext{
${ }^{8}$ When using the double exponential Lévy process, the simulations based on the introduction of jumps in the index dynamics do not significantly modify the ranking of these three strategies. The empirical analysis confirms the results obtained from the simulations.
} 
It implies:

$$
S_{t}=S_{0} \exp \left[\left(\mu-\frac{1}{2} \sigma^{2}\right) t+\sigma W_{t}\right]
$$

Under the (unique) risk neutral probability measure $\mathbb{Q}$, we have $S_{t}=S_{0} \exp \left[\left(r-\frac{1}{2} \sigma^{2}\right) t+\sigma \widetilde{W}_{t}\right]$, where $r$ denotes the riskless interest rate and process $\widetilde{W}$ is a Brownian motion with respect to $\mathbb{Q}$. Such an assumption is commonly introduced when dealing with the pricing of structured funds. In what follows, we examine how prices and returns evolve according to various choices of drift $\mu$, interest rate $r$ and volatility $\sigma$.

One of the most straightforward ways to include jumps in the index price dynamics is to add an independent compound Poisson process to the diffusion component. This introduces two additional independent sources of randomness:

- A Poisson process $N$ which is independent from a Brownian motion $W_{J}$ and which models the jumps arrival times $T_{n}$, with intensity $\lambda$ (i.e. the average number of jumps per unit of time). ${ }^{9}$ $N_{t}=\sum_{T_{n} \leq t} 1$ is the process which counts the number of jumps on $[0, t]$.

- A sequence of independent and identically distributed (i.i.d.) random variables that correspond to the random jump sizes.

The dynamics of the index price $S^{J}$ in the presence of jumps is given by:

$$
\frac{d S_{t}^{J}}{S_{t}^{J}}=\mu_{J} d t+\sigma_{J} d W_{J, t}+\delta_{J, t} d N_{t}, \text { with } \delta_{J, T_{n}}=\frac{\Delta S_{T_{n}}^{J}}{S_{T_{n-}}^{J}},
$$

where the sequence of relative jumps $\left(\Delta S_{T_{n}}^{J} / S_{T_{n-}}^{J}\right)_{n}$ is a sequence of i.i.d. random variables.

We deduce:

$$
S_{t}^{J}=S_{0}^{J} \exp \left[\left(\mu_{J}-\frac{\sigma_{J}^{2}}{2}\right) t+\sigma_{J} W_{J, t}+\sum_{T_{n} \leq t} \ln \left(1+\delta_{J, T_{n}}\right)\right] .
$$

We denote by $b$ the common expectation of the relative jumps and by $c$ the expectation of their squares. Expectation and variance of the risky asset return are given by:

$$
\left\{\begin{array}{l}
E\left[S_{t}^{J} / S_{0}^{J}\right]=e^{\left[\mu_{J}+b \lambda\right] t} \\
\operatorname{Var}\left[S_{t}^{J} / S_{0}^{J}\right]=e^{2\left[\mu_{J}+b \lambda\right] t}\left[e^{\left(\sigma_{J}^{2}+c \lambda\right) t}-1\right]
\end{array}\right.
$$

In what follows, we consider the double exponential Lévy process. This kind of dynamics was applied to option pricing by Kou and Wang (2004) and has been widely used since (see e.g. Bertrand and Prigent, 2011). It is based on a Brownian component with drift and a jump component modeled by a compound Poisson process with jump sizes having a double exponential distribution. In this case, the risky asset price follows the following stochastic differential equation (SDE):

$$
\frac{d S_{t}}{S_{t}}=\mu_{J} d t+\sigma_{J} d W_{J, t}+d\left(\sum_{n=1}^{N_{t}}\left(\frac{\Delta S_{T_{n}}}{S_{T_{n}}}\right)\right),
$$

\footnotetext{
${ }^{9}$ Note that, since most of these financial products are based on the monthly or quarterly returns of the benchmark risky asset, their intraday jumps have no direct impact on the fund's return. Thus we do not introduce a jump process with infinite intensity of jumps.
} 
where the relative jump sizes $\frac{\Delta S_{T_{n}}}{S_{T_{n}}}$, with values in $]-1, \infty\left[\right.$, are i.i.d. variables such that $Z_{n}=$ $\ln \left(1+\frac{\Delta S_{T_{n}}}{S_{T_{n}}}\right)$ has an asymmetrical double exponential distribution with a probability density function (pdf) given by:

$$
f_{Z}(z)=p \cdot \eta_{1} e^{-\eta_{1} z} \mathbb{I}_{\{z \geq 0\}}+q \cdot \eta_{2} e^{\eta_{2} z} \mathbb{I}_{\{z<0\}}, \eta_{1}>1, \eta_{2}>0,
$$

where $p, q \geq 0$ are respectively equal to the probability of an upside jump and a downside jump $(p+q=1)$. It means that:

$$
\ln \left(1+\frac{\Delta S_{T_{n}}}{S_{T_{n}}}\right)=Z_{n}=\left\{\begin{array}{c}
\xi^{+}, \text {with probability } p \\
-\xi^{-}, \text {with probability } q
\end{array},\right.
$$

where $\xi^{+}$and $\xi^{-}$are exponential random variables with respective means $\frac{1}{\eta_{1}}$ and $\frac{1}{\eta_{2}}$. Note that the processes $\left(N_{t}\right)_{t},\left(W_{J, t}\right)_{t}$ and the sequence $\left(Z_{n}\right)_{n}$ are assumed to be independent. The solution of the SDE (13) is given by:

$$
S_{t}=S_{0} \cdot \exp \left[\left(\mu_{J}-\frac{1}{2} \sigma_{J}^{2}\right) t+\sigma_{J} W_{J, t}+\sum_{n=1}^{N_{t}} Z_{n}\right] .
$$

Note that we have:

$$
\mathbb{E}\left[Z_{n}\right]=\frac{p}{\eta_{1}}-\frac{q}{\eta_{2}}, \mathbb{V}\left[Z_{n}\right]=p q\left(\frac{1}{\eta_{1}}-\frac{1}{\eta_{2}}\right)^{2}+\left(\frac{p}{\eta_{1}^{2}}-\frac{q}{\eta_{2}^{2}}\right)
$$

and:

$$
\mathbb{E}\left[\frac{\Delta S_{T_{n}}}{S_{T_{n}}}\right]=\mathbb{E}\left[e^{Z_{n}}\right]-1=q \frac{\eta_{2}}{\eta_{2}+1}+p \frac{\eta_{1}}{\eta_{1}-1}-1, \eta_{1}>1, \eta_{2}>0
$$

Condition $\eta_{1}>1$ is necessary to ensure that $\mathbb{E}\left[\frac{\Delta S_{T_{n}}}{S_{T_{n}}}\right]<\infty$ and $\mathbb{E}\left[S_{t}\right]<\infty .{ }^{10}$ Denote by $X_{t}$ the discounted process of the log returns of $S_{t}$. We have:

$$
X_{t}=\ln \left(\frac{S_{t}}{S_{0}}\right)-r t=\left(\mu_{J}-r-\frac{1}{2} \sigma_{J}^{2}\right) t+\sigma_{J} W_{J, t}+\sum_{n=1}^{N_{t}} Z_{n} .
$$

In what follows, we examine the pricing of structured products in such a dynamic framework.

\section{Valuation of "Asian" fund.}

To obtain the value of parameter $\alpha_{a s}$, we need to calculate the value of the Asian call, namely Call $^{\text {Asian }}\left(T, A(T), S_{0}, r\right)$ (see equation 3 ).

Note that, even with GBM, no exact analytical pricing formula is available for Asian options defined in terms of arithmetic averages. Thus, several analytical approximations have been proposed to approximate the distribution of the average $A(T)=\frac{1}{n T} \sum_{i=1}^{n T} S_{i}$. For example, Turnbull and Wakeman (1991) attempted to approximate the distribution of the arithmetic average (for which no simple specification is known) by a more tractable one. Nevertheless, this approximation does not work very well for long term options. Here, we compute the price of the Asian option by running a Monte Carlo experiment with control variate technique as in Boyle, Broadie and Glasserman (1997).

We use the standard no-arbitrage pricing theory, which implies that the initial value of the Asian call the satisfies:

$$
\operatorname{Call}^{\text {Asian }}\left(T, A(T), S_{0}, r\right)=e^{-r T} \mathbb{E}_{\mathbb{Q}}\left[A(T)-S_{0}\right]^{+},
$$

\footnotetext{
${ }^{10}$ If $Z$ has a Gaussian distribution, we obtain exactly the jump-diffusion model proposed by Merton (1976).
} 
where $\mathbb{Q}$ is a risk-neutral probability. For GBM, the measure $\mathbb{Q}$ is unique. For the jump-diffusion model, given that the market is no longer complete, the measure $\mathbb{Q}$ is no longer unique.

We assume, as in Merton (1976), that the extra randomness due to jumps can be diversified away, which implies that the distributions of jumps under the historical probability and the risk-neutral probability are identical. Therefore, under the risk-neutral probability $\mathbb{Q}$, the dynamics of the risky asset is given by: ${ }^{11}$

$$
S_{t}^{J}=S_{0}^{J} \exp \left[\left(r-b \lambda-\frac{\sigma_{J}^{2}}{2}\right) t+\sigma_{J} \widetilde{W}_{J, t}+\sum_{T_{n} \leq t} Z_{n}\right],
$$

where $\widetilde{W}_{J}$ is a standard Brownian motion under $\mathbb{Q}$.

\section{Valuation of "Average" fund.}

To obtain the value of parameter $\alpha_{a v}$, we need to calculate the value of the Average of calls (see equation 7). The valuation of the "Average of calls" is much easier since it corresponds to a sum of standard European calls with different maturities $i$. Using the same no-arbitrage valuation arguments as previously, we get:

$$
\operatorname{Call}^{\text {Average }}\left(T, S, S_{0}, r\right)=\frac{1}{n T} \sum_{i=1}^{n T} e^{-r(T-i)} e^{-r i} \mathbb{E}_{\mathbb{Q}}\left[\left(S_{i}-S_{0}\right)^{+}\right]
$$

where $e^{-r i} \mathbb{E}_{\mathbb{Q}}\left[\left(S_{i}-S_{0}\right)^{+}\right]=\operatorname{Call}\left(i, S, S_{0}, r\right)$.

Note that Relation (15) involves amounts $\left(S_{i}-S_{0}\right)^{+}$which are not remunerated at the riskless rate $r$ during the remaining time periods $[i, T]$. Indeed, only the amount $\left(S_{i}-S_{0}\right)^{+}$is delivered at global maturity $T$.

\section{Comparison of funds}

Both the Asian and the Average funds provide full insurance on the initial capital $V_{0}$ and both enable fluctuations of the underlying risky asset to be smoothed, but via different methods: for the Asian fund, using the excess of the average of periodic returns with respect to the initial risky asset value $S_{0}$; for the Average fund, using the average of the excess of the periodic returns with respect to $S_{0}$. These two funds (or strategies) can thus clearly be compared, since they are competing investment vehicles (using Monte Carlo simulations under the "real-world probability measure"). We also use the OBPI strategy as a benchmark case. ${ }^{12}$

\subsection{Comparison of parameters $\alpha_{a s}$ and $\alpha_{a v}$}

To compare parameters $\alpha_{a s}$ and $\alpha_{a v}$ from Equations (3) and (7), we must compare the price Call Average $\left(T, S, S_{0}, r\right)$ with the price Call $^{\text {Asian }}\left(T, A(T), S_{0}, r\right)$. Applying Jensen's inequality to the convex function $x^{+}=$ $\operatorname{Max}(x, 0)$, we deduce that the payoff of the Average of calls is higher than the payoff of the Asian option:

$$
\frac{1}{n T} \sum_{i=1}^{n T}\left(S_{i}-S_{0}\right)^{+} \geq\left(\frac{1}{n T} \sum_{i=1}^{n T} S_{i}-S_{0}\right)^{+}
$$

\footnotetext{
${ }^{11}$ Applying Relation (12), we get $E_{\mathbb{Q}}\left[S_{t}^{J} / S_{0}^{J}\right]=e^{(r-b \lambda+b \lambda) T}=e^{r T}$, which is a necessary condition since $\mathbb{Q}$ is a risk-neutral probability.

${ }^{12}$ The Option Based Portfolio Insurance (OBPI) is defined in the following way : $V_{T}=V_{0}+\alpha_{O B P I} V_{0}\left[S_{T}-S_{0}\right]^{+}$and $\alpha_{O B P I}$ is such that $V_{0}=e^{-r T}\left[V_{0}+\alpha_{O B P I} V_{0} E_{\mathbb{Q}}\left[S_{T}-S_{0}\right]^{+}\right]$.
} 
Therefore, the price Call $^{\text {Average }}\left(T, S, S_{0}, r\right)$ of the Average of calls is also higher than the price $\operatorname{Call}^{\text {Asian }}\left(T, A(T), S_{0}, r\right)$ of the Asian option. Thus we deduce that $\alpha_{a s} \geq \alpha_{a v}$.

In conclusion, the payoff of the Average of calls is higher but its weight, $\alpha_{a v}$, is smaller than the weight of the Asian payoff, $\alpha_{a s}$. Consequently, the comparison of the payoffs of both corresponding funds is not straightforward even if the initial value, $V_{0}$, of the funds is the same.

\subsection{Performances with respect to different scenarios}

We begin by illustrating the behavior of both strategies as well as the OBPI when some typical paths of the underlying index are followed over six years, for an initial fund value of $100 .{ }^{13}$

First, in a bullish market, both path-dependent strategies capture part of the increase of the risky reference asset $S$ ("the market"). The Asian-type strategy performs slightly better than the Average strategy, as shown in Figure (1.A). In this case, the OBPI is the best strategy because it does not depend on an average, but only on the terminal value of the underlying asset.

Remark 1 If for all maturities $i$, we have $S_{i} \geq S_{0}$, then the Asian option and the Average of calls provide the same payoff:

$$
\frac{1}{n T} \sum_{i=1}^{n T}\left(S_{i}-S_{0}\right)^{+}=\frac{1}{n T} \sum_{i=1}^{n T} S_{i}-S_{0}=\left(\frac{1}{n T} \sum_{i=1}^{n T} S_{i}-S_{0}\right)^{+}
$$

while the OBPI fund satisfies: $\left(S_{T}-S_{0}\right)^{+}=S_{T}-S_{0}$.

Note also that, if for all maturities $i, S_{T} \geq S_{i}$, then we get: $S_{T}>\frac{1}{n T} \sum_{i=1}^{n T} S_{i}$. It means that OBPI payoff is higher than the others.

This case corresponds to Figure 1.A. The Asian option and Average of calls payoff are equal but since $\alpha_{a s} \geq \alpha_{a v}$, the payoff of the Asian fund is higher than the payoff of the Average fund.

Note also that, if the price process $S$ is increasing along the whole management period, then the two previous conditions, namely: for all $i, S_{i} \geq S_{0}$ and $S_{T} \geq S_{i}$, are satisfied.

Remark 2 If for all maturities $i$, we have $S_{i} \leq S_{0}$, then all the products provide exactly the guaranteed amount $V_{0}$, since we have: $\frac{1}{n T} \sum_{i=1}^{n T}\left(S_{i}-S_{0}\right)^{+}=\left(\frac{1}{n T} \sum_{i=1}^{n T} S_{i}-S_{0}\right)^{+}=\left(S_{T}-S_{0}\right)^{+}=0$. When, at the end of the management period a net market downturn has occurred, all three strategies merely deliver the guaranteed capital (see Figure 1.B).

Another pattern that is commonly encountered in financial markets is when the market is first bullish and then bearish, with a terminal value below the starting one. In such a case, the Average strategy takes advantage of its "call feature at each date", which prevents values below the initial value from being taken into account when computing the average. Accordingly, it performs better than the Asian strategy, for which values below the initial value are taken into account in the calculation of the average.

The OBPI winds up at the guaranteed value, since it only depends on the terminal value of the underlying asset (see Figure 1.C). The market can also be first bearish then bullish, with a final value above the initial one. Again, the Average strategy performs better than the Asian one for the same reasons but the OBPI is the best strategy (see Figure 1.D).

\footnotetext{
${ }^{13}$ Note that not all these paths have the same probability of occurrence. We consider the GBM case to determine the coefficients $\alpha_{a s}, \alpha_{a v}$ and $\alpha_{O B P I}$.
} 


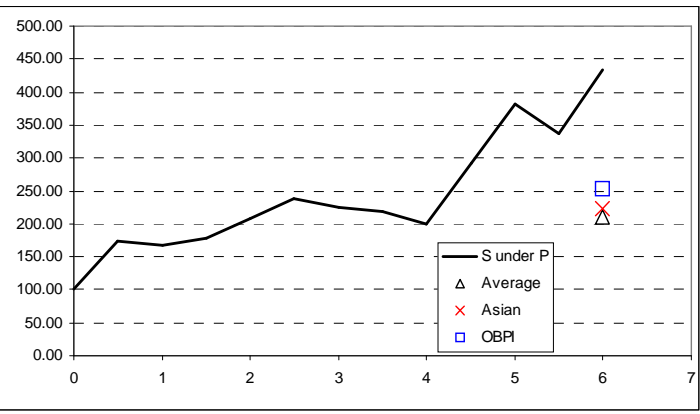

A. Bullish Market

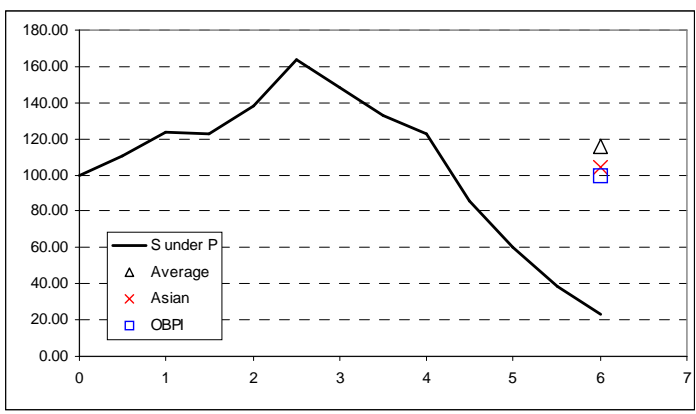

C. Bullish then Bearish Market

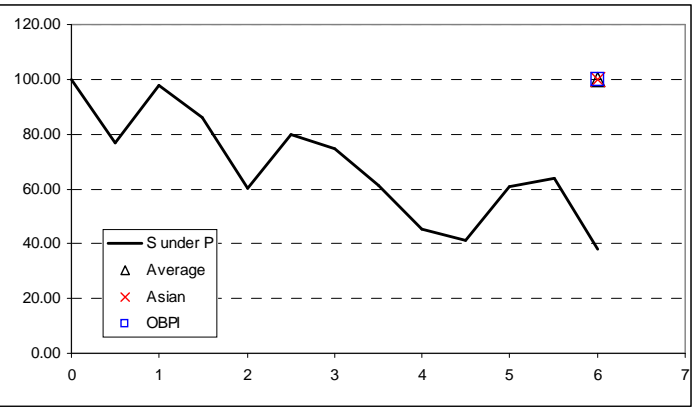

B. Bearish Market

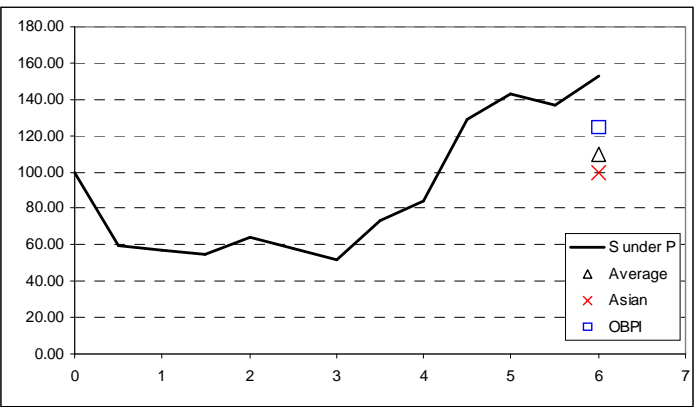

D. Bearish then Bullish Market

Figure 1: Fund performances for different scenarios

\subsection{First four moments}

We now examine the first four moments of both Asian and Average strategies with GBM. Using Monte Carlo simulations ${ }^{14}$, we obtain the rate of return of these strategies over the whole period for various values of the financial market parameters ${ }^{15}(\mu, \sigma)$ and of the length of the management period, $T$. More specifically, we consider that the continuous-time annual expected return $\mu$ of the index varies from $5 \%$ to $10 \%$ and that the continuous-time annual volatility varies from $15 \%$ to $40 \%$. The average in both products is computed on a quarterly basis which is the most common case. The maturity of the structured products ranges from 4 to 14 years. After running Monte Carlo simulations, we obtain that the return of both strategies is increasing in the expected return of the underlying index and decreasing in the volatility of the return of the underlying index. Regarding the standard deviation of the return of both strategies, it is increasing in both volatility and expected return of the underlying index. Thus, a rise in the volatility of the underlying index has a negative impact on both performance and risk of the strategies. For instance, for the Average strategy, ${ }^{16}$ given an annual expected return of $7 \%$ for the underlying index and a time to maturity of 8 years, an increase in its annual volatility from $15 \%$ to $40 \%$ is accompanied by a decrease in expected return from $51.14 \%$ to $39.04 \%$, while at the same time its standard deviation increases from $45.51 \%$ to $75.42 \% .{ }^{17}$ Figures (2.A) and (2.C) show respectively the expected return and the standard

\footnotetext{
${ }^{14}$ Although the initial value of the Average fund can be derived analytically, for consistency with the Asian strategy, we also use simulations.

${ }^{15}$ The level of the risk-free interest rate is constant at $3 \%$.

${ }^{16}$ For the structured funds, both expected return and standard deviation are computed for the whole management period (here 8 years).

${ }^{17}$ The same applies to the Asian strategy, with a decrease in the expected return from $53.47 \%$ to $40.33 \%$ and an increase in the standard deviation from $50.26 \%$ to $84.74 \%$.
} 


\section{A. Expected return of Average Fund}

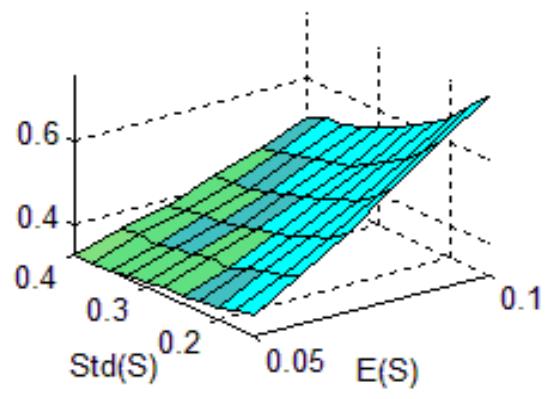

C. Volatility of Average Fund

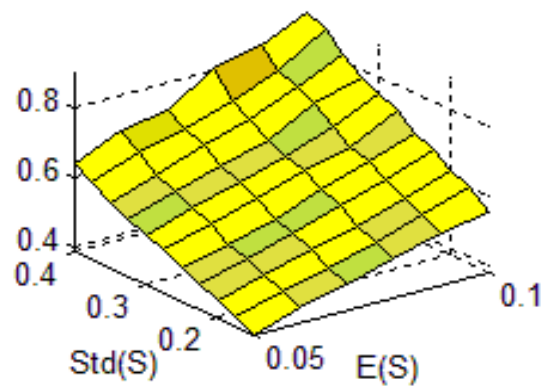

\section{B. Expected return of Asian Fund}

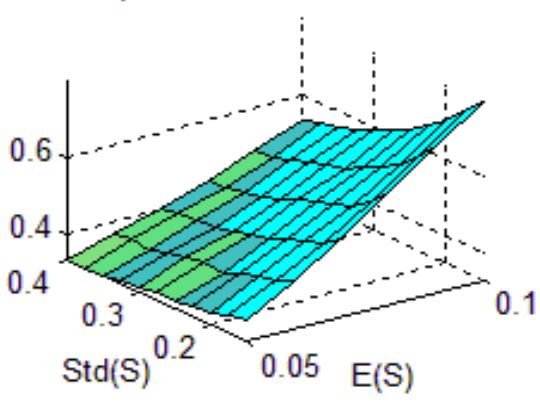

D. Volatility of Asian Fund

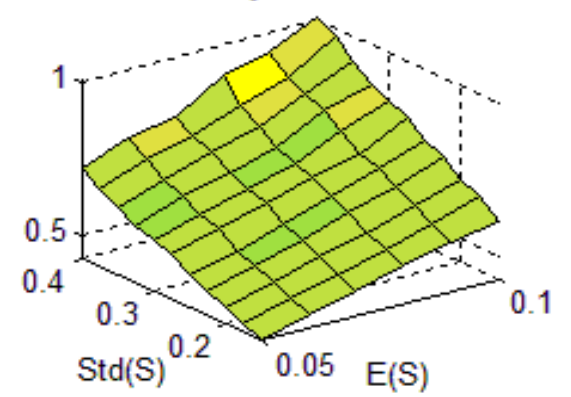

Figure 2: Expected Returns and Volatilities

deviation of the Average fund for $T=8$ as a function of $(\mu, \sigma)$. Figures (2.B) and (2.D) show respectively the expected return and the standard deviation of the Asian fund for $T=8$ as a function of $(\mu, \sigma)$.

If we consider only the first two moments of the strategies (i.e. the expected return and the risk), we note that the Average fund always delivers less expected return and less standard deviation than the Asian fund. Thus, there is no mean-variance dominance between these two funds, as shown in Table 1 and Table 2 for $T=8$.

Table 1: Standard deviation of Average and Asian funds, $\mathrm{T}=8$

\begin{tabular}{ccccccccccc}
\hline & \multicolumn{2}{c}{$\mu=5.00 \%$} & \multicolumn{2}{c}{$\mu=6.00 \%$} & \multicolumn{2}{c}{$\mu=7.00 \%$} & \multicolumn{2}{c}{$\mu=8.00 \%$} & \multicolumn{2}{c}{$\mu=9.00 \%$} \\
\cline { 2 - 10 }$\sigma$ & Aver. & Asian & Aver. & Asian & Aver. & Asian & Aver. & Asian & Aver. & Asian \\
\hline $15 \%$ & $38.32 \%$ & $42.38 \%$ & $42.19 \%$ & $46.64 \%$ & $45.51 \%$ & $50.26 \%$ & $49.03 \%$ & $54.02 \%$ & $52.51 \%$ & $57.74 \%$ \\
$20 \%$ & $42.99 \%$ & $47.93 \%$ & $46.94 \%$ & $52.36 \%$ & $50.02 \%$ & $55.70 \%$ & $54.99 \%$ & $61.18 \%$ & $58.52 \%$ & $65.01 \%$ \\
$25 \%$ & $47.94 \%$ & $53.73 \%$ & $52.01 \%$ & $58.28 \%$ & $54.81 \%$ & $61.39 \%$ & $60.68 \%$ & $67.94 \%$ & $64.98 \%$ & $72.72 \%$ \\
$30 \%$ & $52.44 \%$ & $58.94 \%$ & $55.69 \%$ & $62.54 \%$ & $60.34 \%$ & $67.68 \%$ & $66.99 \%$ & $75.23 \%$ & $70.00 \%$ & $78.41 \%$ \\
$35 \%$ & $57.44 \%$ & $64.48 \%$ & $61.80 \%$ & $69.57 \%$ & $66.82 \%$ & $75.02 \%$ & $70.44 \%$ & $79.13 \%$ & $77.74 \%$ & $87.40 \%$ \\
$40 \%$ & $63.27 \%$ & $71.26 \%$ & $68.49 \%$ & $77.03 \%$ & $75.42 \%$ & $84.74 \%$ & $78.49 \%$ & $88.19 \%$ & $82.97 \%$ & $93.19 \%$ \\
\hline
\end{tabular}

Now also taking account of the OBPI strategy, Table 3 provides the first four moments together with the semi-standard deviation of both fund returns, with respect to the following parametrization 
Table 2: Expected return of Average and Asian funds, $\mathrm{T}=8$

\begin{tabular}{rcccccccccc}
\hline & \multicolumn{2}{c}{$\mu=5.00 \%$} & \multicolumn{2}{c}{$\mu=6.00 \%$} & \multicolumn{2}{c}{$\mu=7.00 \%$} & \multicolumn{2}{c}{$\mu=8.00 \%$} & \multicolumn{2}{c}{$\mu=9.00 \%$} \\
\cline { 2 - 10 }$\sigma$ & Aver. & Asian & Aver. & Asian & Aver. & Asian & Aver. & Asian & Aver. & Asian \\
\hline $15 \%$ & $37.85 \%$ & $38.85 \%$ & $44.36 \%$ & $46.03 \%$ & $51.14 \%$ & $53.47 \%$ & $58.81 \%$ & $61.87 \%$ & $66.70 \%$ & $70.57 \%$ \\
$20 \%$ & $35.51 \%$ & $36.38 \%$ & $40.82 \%$ & $42.23 \%$ & $45.99 \%$ & $47.90 \%$ & $52.56 \%$ & $55.21 \%$ & $58.98 \%$ & $62.31 \%$ \\
$25 \%$ & $34.48 \%$ & $35.25 \%$ & $38.89 \%$ & $40.13 \%$ & $43.00 \%$ & $44.72 \%$ & $49.00 \%$ & $51.41 \%$ & $54.52 \%$ & $57.54 \%$ \\
$30 \%$ & $33.67 \%$ & $34.38 \%$ & $37.16 \%$ & $38.25 \%$ & $40.86 \%$ & $42.36 \%$ & $46.46 \%$ & $48.65 \%$ & $50.26 \%$ & $52.80 \%$ \\
$35 \%$ & $32.54 \%$ & $33.10 \%$ & $36.51 \%$ & $37.53 \%$ & $39.66 \%$ & $41.03 \%$ & $43.51 \%$ & $45.32 \%$ & $49.02 \%$ & $51.53 \%$ \\
$40 \%$ & $32.52 \%$ & $33.12 \%$ & $35.57 \%$ & $36.49 \%$ & $39.04 \%$ & $40.33 \%$ & $41.63 \%$ & $43.28 \%$ & $45.92 \%$ & $48.00 \%$ \\
\hline
\end{tabular}

of the financial market : $\sigma=30 \%, \mu=8 \%, r=3 \%, T=8$. The coefficients alpha are: $\alpha_{a v}=0.8528$, $\alpha_{a s}=0.9428$ and $\alpha_{O B P I}=0.5148$. Table 3 shows that neither strategy dominates the other. The Average fund has less expected return, smaller standard deviation as well as semi-standard deviation, a little more skewness and a little less kurtosis that the Asian fund. Thus, with respect to the first four moments of their returns, there is no clear dominance by one strategy over the other, as illustrated by the last three columns of Table 3, when pairs of strategies are compared. Additionally, the Average and the Asian funds (the two sophisticated funds) are more similar to one another than to the OBPI fund. Results for the OBPI fund are more extreme. ${ }^{18}$

Table 3: First four Moments of Average, Asian and OBPI portfolios

\begin{tabular}{lcccccc}
\hline & Aver. & Asian & OBPI & Av. vs Asian & Av. vs OBPI & Asian vs OBPI \\
\cline { 2 - 7 } Expectation (\%) & $46.46 \%$ & $48.65 \%$ & $54.87 \%$ & - & - & - \\
Standard deviation (\%) & $66.99 \%$ & $75.23 \%$ & $97.81 \%$ & + & + & + \\
Skewness & 3.20 & 3.14 & 4.30 & + & - & - \\
Kurtosis & 20.78 & 20.06 & 36.67 & - & + & + \\
\hline
\end{tabular}

Remark 3 Sensitivity to the volatility of the benchmark index can also be analyzed by computing the Vega of each fund. More generally, from a dynamic point of view, all Greeks of the three funds can be analyzed. Assuming GBM dynamics, with OBPI we simply use the well-known results on Greeks of a standard call option. For the Average fund, Greeks are simply equal to the averages of each respective Greek, which corresponds to the standard Greeks of call options. For example, since the value of the optional component of the Average fund is given by:

$$
e^{-r T} E_{\mathbb{Q}}\left[\frac{1}{n T} \sum_{i=1}^{n T}\left(S_{i}-S_{0}\right)^{+}\right]=\frac{1}{n T} \sum_{i=1}^{n T} e^{-r(T-i)} C\left(S_{0}, S_{0}, d, r, \sigma, i\right),
$$

we deduce the Vega of the Average fund:

$$
\frac{\partial}{\partial \sigma} e^{-r T} \mathbb{E}_{\mathbb{Q}}\left[\frac{1}{n T} \sum_{i=1}^{n T}\left(S_{i}-S_{0}\right)^{+}\right]=\frac{1}{n T} \sum_{i=1}^{n T} e^{-r(T-i)} S_{0} \sqrt{i} \exp \left[-d_{1}(i)^{2} / 2\right] / \sqrt{2 \pi},
$$

\footnotetext{
${ }^{18}$ In Table 3, symbol "+" (resp. "-") corresponds to a better (resp. worse) ranking for the given criterion.
} 
with

$$
d_{1}(i)=\frac{\ln S_{0}-\ln K+\left(r-d+\sigma^{2} / 2\right) i}{\sigma \sqrt{i}} .
$$

For Asian funds, the determination of Greeks is much more involved. They can be computed only by using numerical approximations. For example, Den Iseger and Oldenkamp (2006) provide a stable pricing algorithm which enables both the price and its Greeks to be computed for both lognormal and jump-diffusion processes. It is based on Laplace inversion methods. All densities needed for the computation of the price and the Greeks are computed recursively at the sample times.

\subsection{Cumulative distribution function (CDF) of the three portfolio strategies}

Figure (3.A) illustrates the cumulative distribution function (CDF) of the three fund returns on the whole management period for the same parametrization of the financial market : $\sigma=30 \%, \mu=8 \%, r=3 \%$, $T=8$. Figure (3.B) shows another parametrization of the financial market : $\sigma=25 \%, \mu=6 \%, r=3 \%$, $T=8$.

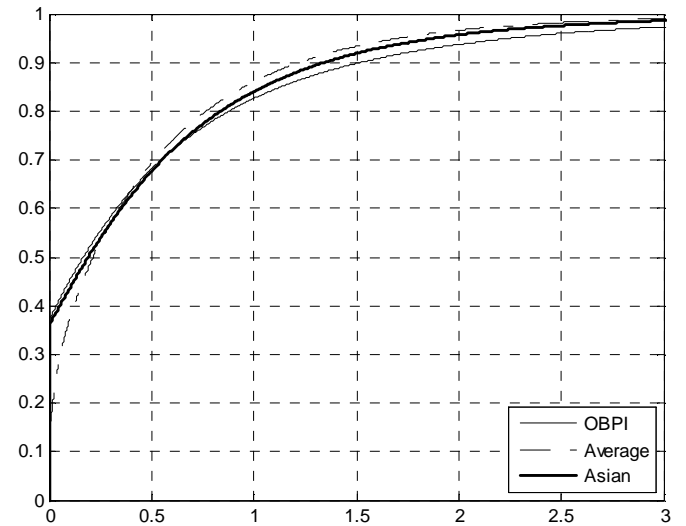

A.

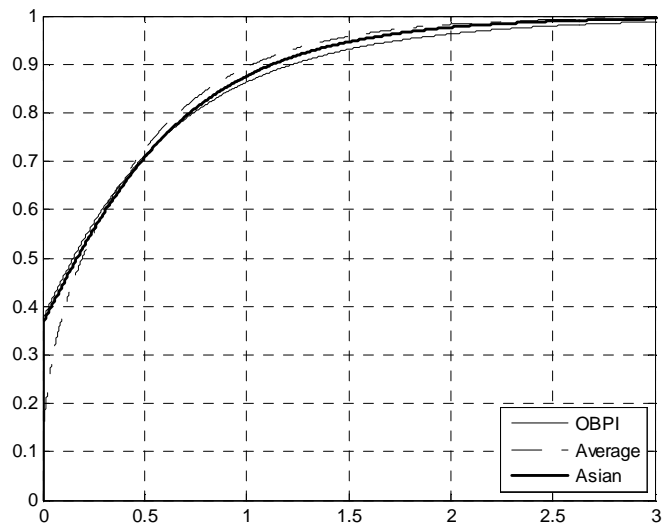

B.

Figure 3: CDF of the Average, the Asian and the OBPI funds

From Figure (3.A) and (3.B), we note that the Average fund has a much lower probability than the two other funds of winding up at the insured amount $V_{0}$ (as can be seen in left part of the figures for return equal to $0 \%$ ). This feature is detailed in the next section.

This highlights the good smoothing properties of the Average fund and points to the fact that the Asian fund in this respect is much more similar to the OBPI fund than to the Average fund. For small returns, the Asian fund is similar to the OBPI fund, while for high returns, the Asian fund becomes similar to the Average fund. 


\subsection{Comparison of probabilities of delivering merely the guaranteed amount}

For a structured fund providing a guarantee corresponding to $100 \%$ of the initial investment, it would be a drawback to deliver merely this guaranteed amount at maturity, whereas simply investing in the safe asset would have provided for example, $20 \%$ over six years. Thus, the probability of delivering merely the guaranteed amount has important implications.

The result of previous section illustrated in Figure (3) is robust, even for continuous-time monitoring (see Appendix for a detailed analysis of the probability of producing merely the guaranteed amount for (theoretical) continuous-time monitoring).

Proposition 4 Whatever the market fluctuations, the payoff of the Average fund always has the lowest probability of being merely equal to the guaranteed amount $V_{0}$.

Proof. - First, we compare the probability of obtaining only the initial value at maturity for the Average and the OBPI funds. We note that:

$$
\mathbb{P}\left[\frac{1}{n T} \sum_{i=1}^{n T}\left(S_{i}-S_{0}\right)^{+}>0\right]=\mathbb{P}\left[\exists i \in\{1, \ldots, n T\}, S_{i}>S_{0}\right]
$$

Since we have $\mathbb{P}\left[\exists i \in\{1, \ldots, n T\}, S_{i}>S_{0}\right] \geq \mathbb{P}\left[S_{T}>S_{0}\right]$ (in this latter case, $i=n T$ ), we deduce that $\mathbb{P}\left[V_{T}^{\text {Average }}>V_{0}\right] \geq \mathbb{P}\left[V_{T}^{O B P I}>V_{0}\right]$ and consequently, $\mathbb{P}\left[V_{T}^{\text {Average }}=V_{0}\right] \leq \mathbb{P}\left[V_{T}^{O B P I}=V_{0}\right]$.

- Second, we compare this probability for the Average and the Asian funds. Applying Jensen's inequality, we deduce:

$$
\frac{1}{n T} \sum_{i=1}^{n T}\left(S_{i}-S_{0}\right)^{+} \geq\left(\frac{1}{n T} \sum_{i=1}^{n T} S_{i}-S_{0}\right)^{+}
$$

which implies:

$$
\mathbb{P}\left[\frac{1}{n T} \sum_{i=1}^{n T}\left(S_{i}-S_{0}\right)^{+}>0\right] \geq \mathbb{P}\left[\left(\frac{1}{n T} \sum_{i=1}^{n T} S_{i}-S_{0}\right)^{+}>0\right] .
$$

This latter relation proves that $\mathbb{P}\left[V_{T}^{\text {Average }}>V_{0}\right] \geq \mathbb{P}\left[V_{T}^{\text {Asian }}>V_{0}\right]$. Thus, we get:

$$
\mathbb{P}\left[V_{T}^{\text {Average }}=V_{0}\right] \leq \mathbb{P}\left[V_{T}^{\text {Asian }}=V_{0}\right] .
$$

As shown in Tables 4 and 5, for the Asian fund, the probability of obtaining merely the guarantee at maturity is quite close to that of the OBPI fund (slightly higher). For most cases, it lies between approximately $15 \%$ and $40 \%$, which is much higher than the corresponding probability for the Average fund and is significantly disadvantageous for these two funds. 
Table 4 shows this probability for various drift and volatility values (for $T=8$ years and $r=3 \%$ ). As expected, this probability is decreasing with respect to the drift and increasing with respect to the volatility.

Table 4: Probability of obtaining merely the insured amount as a function of $\mu$ and $\sigma$

\begin{tabular}{|c|c|c|c|c|c|c|c|c|c|c|c|c|}
\hline \multirow[b]{2}{*}{$\sigma$} & \multicolumn{3}{|c|}{$\mu=10 \%$} & \multicolumn{3}{|c|}{$\mu=9 \%$} & \multicolumn{3}{|c|}{$\mu=8 \%$} & \multicolumn{3}{|c|}{$\mu=7 \%$} \\
\hline & Aver. & Asian & OBPI & Aver. & Asian & OBPI & Aver. & Asian & OBPI & Aver. & Asian & OBPI \\
\hline $20.0 \%$ & $1.82 \%$ & $14.92 \%$ & $12.88 \%$ & $2.32 \%$ & $18.03 \%$ & $16.19 \%$ & $3.01 \%$ & $21.43 \%$ & $19.79 \%$ & $3.75 \%$ & $25.25 \%$ & $23.97 \%$ \\
\hline $25.0 \%$ & $3.38 \%$ & $22.83 \%$ & $21.75 \%$ & $4.07 \%$ & $26.07 \%$ & $25.30 \%$ & $4.76 \%$ & $29.26 \%$ & $28.96 \%$ & $5.67 \%$ & $32.88 \%$ & $33.17 \%$ \\
\hline $30.0 \%$ & $5.02 \%$ & $29.93 \%$ & $30.19 \%$ & $5.77 \%$ & $32.93 \%$ & $33.59 \%$ & $6.55 \%$ & $35.88 \%$ & $36.90 \%$ & $7.47 \%$ & $38.96 \%$ & $40.61 \%$ \\
\hline $35.0 \%$ & $6.70 \%$ & $35.85 \%$ & $37.56 \%$ & $7.54 \%$ & $38.70 \%$ & $40.90 \%$ & $8.24 \%$ & $41.39 \%$ & $43.96 \%$ & $9.12 \%$ & $44.19 \%$ & $47.20 \%$ \\
\hline $40.0 \%$ & $8.39 \%$ & $41.31 \%$ & $44.37 \%$ & $9.09 \%$ & $43.68 \%$ & $47.08 \%$ & $9.94 \%$ & $46.28 \%$ & $50.10 \%$ & $10.75 \%$ & $48.52 \%$ & $52.70 \%$ \\
\hline
\end{tabular}

Table 5 illustrates this probability as a function of the investment horizon $T$ (for $\mu=10 \%, \sigma=25 \%$ and $r=3 \%$ ). Again, as expected, this probability decreases as the horizon increases.

Table 5: Probability of obtaining merely the insured amount as a function of $\mathrm{T}$

\begin{tabular}{ccccccccc}
\hline $\mathrm{T}$ & 1 & 2 & 3 & 4 & 5 & 6 & 7 & 8 \\
\hline Average & $20.31 \%$ & $12.40 \%$ & $8.89 \%$ & $6.86 \%$ & $5.57 \%$ & $4.56 \%$ & $3.91 \%$ & $3.38 \%$ \\
Asian & $39.22 \%$ & $35.43 \%$ & $32.26 \%$ & $29.87 \%$ & $27.86 \%$ & $25.97 \%$ & $24.35 \%$ & $22.92 \%$ \\
OBPI & $39.16 \%$ & $34.89 \%$ & $31.58 \%$ & $28.94 \%$ & $26.95 \%$ & $24.97 \%$ & $23.30 \%$ & $21.86 \%$ \\
\hline
\end{tabular}

Table 6 shows the effect of computing the average on more frequent data than quarterly data, namely on weekly data.

Only, the Average fund is significantly affected by this effect. Its probability of delivering merely the guaranteed amount decreases with the frequency of the data and converges to 0 as soon as this frequency rises.

An analytical proof of this result for the continuous-time monitoring case ("the limit case") is available in the Appendix.

Table 6: Probability of obtaining merely the insured amount as a function of $\mathrm{T}$ (weekly data)

\begin{tabular}{ccccccccc}
\hline $\mathrm{T}$ & 1 & 2 & 3 & 4 & 5 & 6 & 7 & 8 \\
\hline Average & $5.38 \%$ & $3.25 \%$ & $2.38 \%$ & $1.86 \%$ & $1.42 \%$ & $1.18 \%$ & $1.04 \%$ & $0.95 \%$ \\
Asian & $39.51 \%$ & $35.73 \%$ & $32.37 \%$ & $29.99 \%$ & $28.15 \%$ & $26.15 \%$ & $24.66 \%$ & $22.94 \%$ \\
OBPI & $39.13 \%$ & $34.80 \%$ & $31.63 \%$ & $29.23 \%$ & $26.95 \%$ & $24.95 \%$ & $23.48 \%$ & $21.83 \%$ \\
\hline
\end{tabular}




\subsection{Comparison by means of Kappa performance measures}

In what follows, we use the Kappa measures introduced by Kaplan and Knowles (2004) to analyze and compare performances of financial structured products.

The Kappa measures involve downside risk measures, and are defined by:

$$
\text { For } l=1,2, \ldots, \operatorname{Kappa}_{l}(L)=\frac{\mathbb{E}_{\mathbb{P}}[X]-L}{\left(\mathbb{E}_{\mathbb{P}}\left[\left[(L-X)^{+}\right]^{l}\right]\right)^{\frac{1}{l}}} .
$$

Note that, when $l=1$, the Kappa measure corresponds to the Sharpe Omega measure and when $l=2$, to the Sortino ratio. Zakamouline (2013) also proves that Kappa performance measures correspond to measures based on piecewise linear plus power utility functions. When $l=3$, the risk measure in the denominator of the Kappa looks like a semi-skewness and when $l=4$, the risk measure in the denominator of the Kappa looks like a semi-kurtosis.

As in Bertrand and Prigent (2011), we examine the choice of the threshold $L$ involved in the Kappa ratios. The threshold $L$ must be determined exogenously, for example with respect to decision criteria (e.g. risk aversion). Unser (2000) emphasizes that individuals often exhibit aversion towards downside risk (values of outcomes lower than a given level). Recall that the initial investment $V_{0}$ is split into two components: the first, equal to $V_{0} e^{-r T}$, corresponds to the investment on the riskless asset; the second, equal to $\left(1-e^{-r T}\right) V_{0}$, is invested in the risky component of the fund with return denoted by $R_{T}^{\text {risky }}$.

Therefore, the expectation of the fund's return is given by:

$$
\mathbb{E}_{\mathbb{P}}\left[\frac{V_{T}}{V_{0}}\right]=1+\left(1-e^{-r T}\right) \mathbb{E}_{\mathbb{P}}\left[R_{T}^{r i s k y}\right]
$$

Thus, every "rationale" threshold $L$ must be lower than $\left(1+\left(1-e^{-r T}\right) \mathbb{E}_{\mathbb{P}}\left[R_{T}^{\text {risky }}\right]\right) V_{0} \cdot{ }^{19}$

Here, we rely on a set of Kappa performance measures to assess the two mean performance strategies as well as the OBPI strategy. More precisely, we compute the Kappa measures corresponding to powers from one to four. We choose to vary the threshold from $2 \%$ to $48 \%$, thus taking account of all the relevant cases.

Table (7) displays the results of Monte Carlo simulations ${ }^{20}$ for GBM with the following parametrization: $\sigma=25 \%, \mu=8 \%, r=3 \%, T=8$. In this setup, the expectations of the three fund returns are the following:

$$
\mathbb{E}_{\mathbb{P}}\left[V_{T}^{\text {Average }} / V_{0}\right]=49.04 \%<\mathbb{E}_{\mathbb{P}}\left[V_{T}^{\text {Asian }} / V_{0}\right]=51.48 \%<\mathbb{E}_{\mathbb{P}}\left[V_{T}^{\text {OBPI }} / V_{0}\right]=58.15 \% .
$$

\footnotetext{
${ }^{19}$ Recall that, for the standard buy-and-hold strategy corresponding to $V_{T}=V_{0}+q S_{T}$, with $q=\frac{V_{0}\left(1-e^{-r T}\right)}{S_{0}}$, threshold $L$ must be lower than $L \leq\left[1+\left(1-e^{-r T}\right) e^{\mu T}\right] V_{0}$. This latter condition implies that $L \leq \mathbb{E}_{\mathbb{P}}\left[V_{T}\right]$. Otherwise, for example, the Sharpe Omega ratio is increasing with respect to the volatility (see Bertrand and Prigent, 2011).

${ }^{20}$ Underlined in the tables are the highest values among the three funds.
} 
Table 7: Kappas of Average, Asian and OBPI funds, $\mathrm{T}=8$

\begin{tabular}{|c|c|c|c|c|c|c|c|c|c|c|c|c|}
\hline \multirow[b]{2}{*}{$L / V_{0}$} & \multicolumn{3}{|c|}{ Kappa 1} & \multicolumn{3}{|c|}{ Kappa 2} & \multicolumn{3}{|c|}{ Kappa 3} & \multicolumn{3}{|c|}{ Kappa 4} \\
\hline & Aver. & Asian & OBPI & Aver. & Asian & OBPI & Aver. & Asian & OBPI & Aver. & Asian & OBPI \\
\hline $2 \%$ & $\underline{194.19}$ & 80.38 & 92.31 & 72.58 & 44.43 & 50.80 & 51.33 & 36.41 & 41.56 & $\underline{42.86}$ & 32.95 & 37.58 \\
\hline $4 \%$ & $\underline{71.90}$ & 37.57 & 43.28 & $\underline{30.72}$ & 21.12 & 24.25 & $\underline{22.71}$ & 17.38 & 19.94 & $\underline{19.38}$ & 15.76 & 18.06 \\
\hline $6 \%$ & $\underline{39.13}$ & 23.37 & 27.03 & $\underline{18.14}$ & 13.36 & 15.42 & 13.77 & 11.04 & 12.73 & $\underline{11.91}$ & 10.03 & 11.56 \\
\hline $8 \%$ & $\underline{24.90}$ & 16.35 & 18.99 & $\underline{12.26}$ & 9.49 & 11.02 & $\underline{9.49}$ & 7.88 & 9.14 & 8.29 & 7.17 & $\underline{8.31}$ \\
\hline $10 \%$ & $\underline{17.28}$ & 12.18 & 14.22 & $\underline{8.92}$ & 7.18 & 8.38 & $\underline{7.01}$ & 5.98 & 6.98 & 6.17 & 5.45 & $\underline{6.36}$ \\
\hline $12 \%$ & $\underline{12.65}$ & 9.44 & 11.08 & $\underline{6.79}$ & 5.64 & 6.63 & 5.41 & 4.72 & $\underline{5.55}$ & 4.79 & 4.31 & $\underline{5.06}$ \\
\hline $14 \%$ & $\underline{9.60}$ & 7.50 & 8.87 & 5.33 & 4.55 & $\underline{5.39}$ & 4.29 & 3.82 & $\underline{4.53}$ & 3.82 & 3.49 & $\underline{4.14}$ \\
\hline $16 \%$ & 7.47 & 6.08 & 7.25 & 4.28 & 3.74 & $\underline{4.46}$ & 3.48 & 3.15 & $\underline{3.76}$ & 3.11 & 2.88 & $\underline{3.44}$ \\
\hline $18 \%$ & 5.92 & 4.98 & $\underline{6.00}$ & 3.48 & 3.11 & $\underline{3.75}$ & 2.85 & 2.63 & $\underline{3.17}$ & 2.56 & 2.41 & 2.91 \\
\hline $20 \%$ & 4.76 & 4.13 & $\underline{5.02}$ & 2.86 & 2.61 & $\underline{3.18}$ & 2.36 & 2.21 & $\underline{2.70}$ & 2.13 & 2.03 & $\underline{2.48}$ \\
\hline $22 \%$ & 3.85 & 3.44 & $\underline{4.24}$ & 2.37 & 2.20 & $\underline{2.71}$ & 1.97 & 1.87 & $\underline{2.31}$ & 1.78 & 1.72 & $\underline{2.12}$ \\
\hline $24 \%$ & 3.14 & 2.88 & $\underline{3.59}$ & 1.97 & 1.86 & $\underline{2.33}$ & 1.65 & 1.59 & $\underline{1.99}$ & 1.50 & 1.46 & $\underline{1.83}$ \\
\hline $26 \%$ & 2.57 & 2.41 & $\underline{3.06}$ & 1.64 & 1.58 & $\underline{2.01}$ & 1.38 & 1.35 & $\underline{1.72}$ & 1.26 & 1.25 & $\underline{1.59}$ \\
\hline $28 \%$ & 2.10 & 2.02 & $\underline{2.61}$ & 1.37 & 1.34 & $\underline{1.73}$ & 1.16 & 1.15 & 1.49 & 1.06 & 1.06 & $\underline{1.38}$ \\
\hline $30 \%$ & 1.72 & 1.69 & $\underline{2.23}$ & 1.13 & 1.13 & 1.50 & 0.96 & 0.98 & $\underline{1.29}$ & 0.88 & 0.90 & $\underline{1.19}$ \\
\hline $32 \%$ & 1.39 & 1.41 & $\underline{1.91}$ & 0.93 & 0.95 & $\underline{1.29}$ & 0.80 & 0.82 & $\underline{1.12}$ & 0.73 & 0.76 & $\underline{1.04}$ \\
\hline $34 \%$ & 1.12 & 1.16 & $\underline{1.63}$ & 0.76 & 0.80 & $\underline{1.11}$ & 0.65 & 0.69 & $\underline{0.97}$ & 0.60 & 0.64 & $\underline{0.90}$ \\
\hline $36 \%$ & 0.89 & 0.95 & $\underline{1.38}$ & 0.61 & 0.66 & $\underline{0.96}$ & 0.53 & 0.57 & $\underline{0.83}$ & 0.49 & 0.53 & $\underline{0.77}$ \\
\hline $38 \%$ & 0.69 & 0.77 & $\underline{1.17}$ & 0.48 & 0.53 & $\underline{0.82}$ & 0.42 & 0.47 & $\underline{0.71}$ & 0.38 & 0.43 & $\underline{0.66}$ \\
\hline $40 \%$ & 0.52 & 0.60 & $\underline{0.98}$ & 0.36 & 0.43 & $\underline{0.69}$ & 0.32 & 0.37 & $\underline{0.60}$ & 0.29 & 0.35 & $\underline{0.56}$ \\
\hline $42 \%$ & 0.37 & 0.46 & $\underline{0.81}$ & 0.26 & 0.33 & $\underline{0.58}$ & 0.23 & 0.29 & $\underline{0.51}$ & 0.21 & 0.27 & $\underline{0.47}$ \\
\hline $44 \%$ & 0.24 & 0.33 & $\underline{0.67}$ & 0.17 & 0.24 & $\underline{0.48}$ & 0.15 & 0.21 & $\underline{0.42}$ & 0.14 & 0.20 & $\underline{0.39}$ \\
\hline $46 \%$ & 0.12 & 0.22 & $\underline{0.53}$ & 0.09 & 0.16 & $\underline{0.39}$ & 0.08 & 0.14 & $\underline{0.34}$ & 0.07 & 0.13 & $\underline{0.32}$ \\
\hline $48 \%$ & 0.02 & 0.12 & $\underline{0.42}$ & 0.01 & 0.09 & $\underline{0.30}$ & 0.01 & 0.08 & $\underline{0.27}$ & 0.01 & 0.07 & $\underline{0.25}$ \\
\hline
\end{tabular}

Firstly, and perhaps surprisingly, the Asian strategy is never dominant among the three funds analyzed here. Nor is it ever able to dominate the OBPI strategy, the closest to it. These findings are robust and remain true whatever the parametrization used for the financial market. We vary the drift parameter of the stock price index from $6 \%$ to $10 \%$ (the risk free rate is still equal to $3 \%$ ) and its volatility from $15 \%$ to $35 \%$. For all volatilities of the underlying risky market, the dominance of the OBPI strategy over the Average strategy increases with the expected return of the risky market (i.e., $\mu$ ). Indeed, we report a lower threshold beyond which the OBPI strategy Kappa values outstrip those of the Average strategy. On the other hand, for all expected returns, the dominance of the Average strategy over the OBPI strategy is increasing with the volatility of the risky market. Again, the smoothing properties of the Average strategy stand out. It is interesting to analyze the effect of decreasing time to maturity of the funds on the comparison for Kappa measures. 
We also introduce here $T=4$ and $T=6$. Again, we consider a numerical base case for the market parametrization: $\sigma=25 \%, \mu=8 \%, r=3 \%$.

Table 8 gives the results. ${ }^{21}$

Note that, for this parametrization and $T=6$, the expectations of the three fund returns are the following:

$$
\mathbb{E}_{\mathbb{P}}\left[V_{T}^{\text {Average }} / V_{0}\right]=32.55 \%<\mathbb{E}_{\mathbb{P}}\left[V_{T}^{\text {Asian }} / V_{0}\right]=34.03 \%<\mathbb{E}_{\mathbb{P}}\left[V_{T}^{\text {OBPI }} / V_{0}\right]=37.59 \%
$$

Table 8: Kappas of Average, Asian and OBPI funds, $\mathrm{T}=6$

\begin{tabular}{cccccccccccccc}
\hline & \multicolumn{3}{c}{ Kappa 1 } & \multicolumn{3}{c}{ Kappa 2} & \multicolumn{3}{c}{ Kappa 3 } & \multicolumn{3}{c}{ Kappa 4 } \\
\cline { 2 - 12 }$L / V_{0}$ & Aver. & Asian & OBPI & Aver. & Asian & OBPI & Aver. & Asian & OBPI & Aver. & Asian & OBPI \\
\hline $2 \%$ & $\underline{105.21}$ & 48.84 & 54.10 & $\underline{43.25}$ & 28.11 & 31.20 & $\underline{31.59}$ & 23.35 & 25.92 & $\underline{26.82}$ & 21.26 & 23.62 \\
$4 \%$ & $\underline{38.22}$ & 22.20 & 24.71 & $\underline{17.94}$ & 13.04 & 14.56 & $\underline{13.68}$ & 10.89 & 12.17 & $\underline{11.86}$ & 9.94 & 11.11 \\
$6 \%$ & $\underline{20.30}$ & 13.41 & 15.01 & $\underline{10.33}$ & 8.03 & 9.03 & $\underline{8.09}$ & 6.74 & 7.59 & $\underline{7.10}$ & 6.16 & 6.94 \\
$8 \%$ & $\underline{\underline{12.58}}$ & 9.07 & 10.24 & $\underline{6.78}$ & 5.54 & 6.28 & $\underline{5.41}$ & 4.67 & 5.30 & 4.80 & 4.28 & $\underline{4.86}$ \\
$10 \%$ & $\underline{\mathbf{8 . 4 5}}$ & 6.51 & 7.42 & $\underline{4.77}$ & 4.05 & 4.64 & 3.87 & 3.43 & $\underline{3.93}$ & 3.46 & 3.15 & $\underline{3.61}$ \\
$12 \%$ & $\underline{5.95}$ & 4.84 & 5.58 & 3.50 & 3.07 & $\underline{3.55}$ & 2.87 & 2.61 & $\underline{3.02}$ & 2.58 & 2.40 & $\underline{2.78}$ \\
$14 \%$ & $\underline{4.32}$ & 3.68 & 4.30 & 2.62 & 2.37 & $\underline{2.78}$ & 2.18 & 2.02 & $\underline{2.38}$ & 1.97 & 1.87 & $\underline{2.19}$ \\
$16 \%$ & 3.18 & 2.82 & $\underline{3.36}$ & 1.99 & 1.85 & $\underline{2.20}$ & 1.67 & 1.59 & $\underline{1.90}$ & 1.52 & 1.46 & $\underline{1.75}$ \\
$18 \%$ & 2.37 & 2.18 & $\underline{2.64}$ & 1.52 & 1.45 & $\underline{1.76}$ & 1.28 & 1.25 & $\underline{1.52}$ & 1.17 & 1.15 & $\underline{1.41}$ \\
$20 \%$ & 1.75 & 1.68 & $\underline{2.09}$ & 1.15 & 1.13 & $\underline{1.41}$ & 0.98 & 0.98 & $\underline{1.22}$ & 0.90 & 0.91 & $\underline{1.13}$ \\
$22 \%$ & 1.29 & 1.28 & $\underline{1.64}$ & 0.86 & 0.87 & $\underline{1.13}$ & 0.74 & 0.76 & $\underline{0.98}$ & 0.68 & 0.70 & $\underline{0.91}$ \\
$24 \%$ & 0.92 & 0.96 & $\underline{1.29}$ & 0.63 & 0.66 & $\underline{0.89}$ & 0.54 & 0.58 & $\underline{0.78}$ & 0.50 & 0.54 & $\underline{0.73}$ \\
$26 \%$ & 0.63 & 0.69 & $\underline{0.99}$ & 0.44 & 0.49 & $\underline{0.70}$ & 0.38 & 0.43 & $\underline{0.61}$ & 0.35 & 0.40 & $\underline{0.57}$ \\
$28 \%$ & 0.39 & 0.47 & $\underline{0.75}$ & 0.28 & 0.34 & $\underline{0.53}$ & 0.24 & 0.30 & $\underline{0.47}$ & 0.23 & 0.28 & $\underline{0.44}$ \\
$30 \%$ & 0.20 & 0.29 & $\underline{0.54}$ & 0.14 & 0.21 & $\underline{\underline{0.39}}$ & 0.13 & 0.18 & $\underline{0.34}$ & 0.12 & 0.17 & $\underline{0.32}$ \\
$32 \%$ & 0.04 & 0.13 & $\underline{0.37}$ & 0.03 & 0.10 & $\underline{0.27}$ & 0.03 & 0.09 & $\underline{0.24}$ & 0.02 & 0.08 & $\underline{0.22}$ \\
\hline
\end{tabular}

For the previous parametrization and $T=4$, the expectations of the three fund returns are the following:

$$
\mathbb{E}_{\mathbb{P}}\left[V_{T}^{\text {Average }} / V_{0}\right]=19.12 \%<\mathbb{E}_{\mathbb{P}}\left[V_{T}^{\text {Asian }} / V_{0}\right]=19.90 \%<\mathbb{E}_{\mathbb{P}}\left[V_{T}^{\text {OBPI }} / V_{0}\right]=21.40 \% .
$$

As the time to maturity shortens, the Average fund is seen to dominate the OBPI fund (see Table 9). Short maturities do not allow a sufficiently low probability of the final index value being less than its initial value. Thus, the smoothing properties of the Average fund can surpass the call properties at maturity of the OBPI fund. However, for long maturities (i.e. 8 years), it seems that the complexity introduced through Average and Asian funds does not pay. Introducing jumps in the index dynamics does not significantly modify the ranking of these three strategies. The Asian fund is never the dominant fund.

\footnotetext{
${ }^{21}$ As explained previously, we must consider only Kappa measures with thresholds below the expected return of the strategy.
} 
Table 9: Kappas of Average, Asian and OBPI funds, $\mathrm{T}=4$

\begin{tabular}{cccccccccccccc}
\hline & \multicolumn{3}{c}{ Kappa 1 } & \multicolumn{3}{c}{ Kappa 2 } & \multicolumn{3}{c}{ Kappa 3 } & \multicolumn{3}{c}{ Kappa 4 } \\
\cline { 2 - 12 }$L / V_{0}$ & Aver. & Asian & OBPI & Aver. & Asian & OBPI & Aver. & Asian & OBPI & Aver. & Asian & OBPI \\
\hline $2 \%$ & $\underline{45.79}$ & 24.68 & 26.64 & $\underline{21.31}$ & 14.97 & 16.19 & $\underline{\underline{16.23}}$ & 12.64 & 13.68 & $\underline{14.06}$ & 11.60 & 12.57 \\
$4 \%$ & $\underline{\underline{15.80}}$ & 10.51 & 11.46 & $\underline{\underline{8.38}}$ & 6.55 & 7.16 & $\underline{6.66}$ & 5.57 & 6.09 & $\underline{5.89}$ & 5.13 & 5.61 \\
$6 \%$ & $\underline{\mathbf{7 . 8 4}}$ & 5.89 & 6.49 & $\underline{4.51}$ & 3.77 & 4.17 & $\underline{3.68}$ & 3.23 & 3.57 & $\underline{3.30}$ & 2.98 & $\underline{3.30}$ \\
$8 \%$ & $\underline{\underline{4.45}}$ & 3.64 & 4.08 & $\underline{2.71}$ & 2.39 & 2.68 & 2.26 & 2.06 & $\underline{2.31}$ & 2.04 & 1.90 & $\underline{2.14}$ \\
$10 \%$ & 2.67 & 2.34 & $\underline{2.68}$ & 1.71 & 1.57 & $\underline{1.80}$ & 1.44 & 1.36 & $\underline{1.56}$ & 1.31 & 1.26 & $\underline{1.45}$ \\
$12 \%$ & 1.61 & 1.50 & $\underline{1.78}$ & 1.07 & 1.03 & $\underline{1.22}$ & 0.92 & 0.90 & $\underline{1.07}$ & 0.84 & 0.84 & $\underline{0.99}$ \\
$14 \%$ & 0.93 & 0.93 & $\underline{1.16}$ & 0.64 & 0.65 & $\underline{0.82}$ & 0.55 & 0.57 & $\underline{0.72}$ & 0.51 & 0.53 & $\underline{0.67}$ \\
$16 \%$ & 0.47 & 0.52 & $\underline{0.72}$ & 0.33 & 0.37 & $\underline{0.51}$ & 0.29 & 0.33 & $\underline{0.45}$ & 0.27 & 0.31 & $\underline{0.42}$ \\
$18 \%$ & 0.14 & 0.22 & $\underline{0.39}$ & 0.10 & 0.16 & $\underline{0.28}$ & 0.09 & 0.14 & $\underline{0.25}$ & 0.08 & 0.13 & $\underline{0.24}$ \\
\hline
\end{tabular}

In what follows, we illustrate this, using empirical data for the underlying index.

\subsection{Numerical illustration based on empirical analysis}

To illustrate our results, we consider two main financial indices, namely the S\&P 500 and the Dow Jones Euro Stoxx 50 Index. We use weekly data from January 1992 to December 2012 (dividends not adjusted).

For the estimation, we consider the double exponential Lévy process, defined in Section (2.3). As in Ramezani and Zeng (2007), we use the maximum-likelihood method to estimate all the parameters associated with the double exponential Lévy process.

We denote respectively by $\lambda_{1}$ and $\lambda_{2}$ the products $p \lambda$ and $q \lambda_{2}$ where $\lambda$ is the jump intensity. Recall that the expectation of the positive relative jump (resp. negative relative jump) is equal to $\eta_{1}^{-1}$ (resp. $\left.\eta_{2}^{-1}\right)$. The weekly expected return $\mu_{J}$ and the weekly volatility $\sigma_{J}$ are also given in Table 10 .

Table 10: Parameters of the double exponential Lévy process

\begin{tabular}{|l|l|l|l|l|l|l|}
\hline & $\lambda_{1}($ "up") & $\lambda_{2}$ ("down") & $\begin{array}{c}\mu_{J} \\
(" d r i f t ")\end{array}$ & $\begin{array}{c}\sigma_{J} \\
\text { ("vol") }\end{array}$ & $\eta_{1}($ ("up") & $\eta_{2}$ ("down") \\
\hline S\&P 500 & 0.320 & 0.468 & 0.00368 & 0.0137 & 67.135 & 65.880 \\
\hline Euro Stoxx 50 & 0.152 & 0.893 & 0.0109 & 0.0175 & 44.414 & 68.725 \\
\hline
\end{tabular}

Tables 11 and 12 show the Kappa ratios of the three funds for the S\&P 500 and the Euro Stoxx 50 respectively. We fix the time horizon $T$ at eight years and assume that the riskless asset has a return equal to $3 \%$ per year. 
We find the same pattern as in the previous section on Monte Carlo simulations for GBM. The Asian fund is never the best fund, whatever the choice of power for the Kappa measure. It never dominates the OBPI fund. It can be better than the Average fund but only when the $L / V_{0}$ ratio is high. The Average fund outperforms the other for relatively low $L / V_{0}$ ratios and for small powers ( $l=1$ for example).

Table 11: Kappas of Average, Asian and OBPI funds in the presence of jumps: S\&P 500

\begin{tabular}{|c|c|c|c|c|c|c|c|c|c|c|c|c|}
\hline \multirow[b]{2}{*}{$L / V_{0}$} & \multicolumn{3}{|c|}{ Kappa 1} & \multicolumn{3}{|c|}{ Kappa 2} & \multicolumn{3}{|c|}{ Kappa 3} & \multicolumn{3}{|c|}{ Kappa 4} \\
\hline & Aver. & Asian & OBPI & Aver. & Asian & OBPI & Aver. & Asian & OBPI & Aver. & Asian & OBPI \\
\hline $2 \%$ & $\underline{469.0}$ & 172.1 & 219.1 & $\underline{121.9}$ & 70.43 & 84.40 & 76.24 & 52.17 & 61.26 & $\underline{59.83}$ & 44.88 & 52.16 \\
\hline $4 \%$ & $\underline{168.6}$ & 79.98 & 101.7 & $\underline{51.44}$ & 33.54 & 40.31 & $\underline{33.87}$ & 25.01 & 29.47 & $\underline{27.24}$ & 21.56 & 25.16 \\
\hline $6 \%$ & $\underline{89.66}$ & 49.51 & 62.93 & $\underline{30.23}$ & 21.28 & 25.64 & 20.56 & 15.96 & 18.88 & $\underline{16.80}$ & 13.80 & 16.16 \\
\hline $8 \%$ & $\underline{56.04}$ & 34.42 & 43.82 & $\underline{20.38}$ & 15.16 & 18.33 & $\underline{14.20}$ & 11.45 & 13.59 & $\underline{11.74}$ & 9.92 & 11.67 \\
\hline $10 \%$ & $\underline{38.25}$ & 25.52 & 32.53 & $\underline{14.81}$ & 11.51 & 13.96 & $\underline{\underline{10.53}}$ & 8.74 & 10.42 & $\overline{8.79}$ & 7.60 & $\underline{8.98}$ \\
\hline $12 \%$ & $\overline{27.60}$ & 19.69 & 25.13 & $\underline{11.28}$ & 9.09 & 11.07 & $\overline{8.15}$ & 6.94 & $\underline{8.32}$ & 6.86 & 6.05 & $\overline{7.18}$ \\
\hline $14 \%$ & $\underline{20.71}$ & 15.62 & 19.95 & 8.87 & 7.37 & $\underline{9.02}$ & 6.50 & 5.66 & $\underline{6.82}$ & 5.51 & 4.94 & $\underline{5.90}$ \\
\hline $16 \%$ & 15.99 & 12.63 & $\underline{16.16}$ & 7.13 & 6.09 & 7.48 & 5.30 & 4.70 & $\underline{5.70}$ & 4.52 & 4.12 & 4.95 \\
\hline $18 \%$ & 12.60 & 10.36 & $\underline{13.29}$ & 5.83 & 5.10 & $\underline{6.30}$ & 4.39 & 3.96 & $\underline{4.83}$ & 3.76 & 3.48 & $\underline{4.20}$ \\
\hline $20 \%$ & 10.09 & 8.58 & $\underline{11.06}$ & 4.83 & 4.31 & $\overline{5.37}$ & 3.67 & 3.37 & $\underline{4.14}$ & 3.17 & 2.96 & $\overline{3.61}$ \\
\hline $22 \%$ & 8.17 & 7.17 & $\underline{9.29}$ & 4.03 & 3.68 & $\overline{4.61}$ & 3.10 & 2.89 & $\overline{3.57}$ & 2.69 & 2.55 & $\overline{3.13}$ \\
\hline $24 \%$ & 6.68 & 6.03 & $\underline{7.85}$ & 3.39 & 3.15 & $\underline{3.98}$ & 2.63 & 2.49 & $\underline{3.11}$ & 2.29 & 2.20 & $\underline{2.73}$ \\
\hline $26 \%$ & 5.50 & 5.09 & $\overline{6.68}$ & 2.87 & 2.71 & $\overline{3.46}$ & 2.24 & 2.16 & $\overline{2.71}$ & 1.96 & 1.91 & $\overline{2.39}$ \\
\hline $28 \%$ & 4.54 & 4.31 & 5.71 & 2.43 & 2.34 & $\underline{3.01}$ & 1.92 & 1.87 & $\underline{2.38}$ & 1.68 & 1.66 & 2.10 \\
\hline $30 \%$ & 3.77 & 3.65 & $\overline{4.89}$ & 2.06 & 2.02 & $\underline{2.63}$ & 1.64 & 1.62 & $\overline{2.09}$ & 1.44 & 1.44 & $\underline{1.85}$ \\
\hline $32 \%$ & 3.13 & 3.10 & $\overline{4.21}$ & 1.75 & 1.74 & $\overline{2.30}$ & 1.40 & 1.41 & $\overline{1.84}$ & 1.24 & 1.25 & $\underline{1.63}$ \\
\hline $34 \%$ & 2.59 & 2.63 & $\underline{3.62}$ & 1.48 & 1.50 & $\underline{2.02}$ & 1.20 & 1.22 & $\underline{1.62}$ & 1.06 & 1.09 & $\underline{1.44}$ \\
\hline $36 \%$ & 2.14 & 2.22 & $\overline{3.12}$ & 1.25 & 1.29 & $\overline{1.77}$ & 1.01 & 1.05 & $\overline{1.43}$ & 0.90 & 0.94 & $\overline{1.27}$ \\
\hline $38 \%$ & 1.76 & 1.87 & $\underline{2.68}$ & 1.05 & 1.11 & $\underline{1.55}$ & 0.86 & 0.91 & $\underline{1.25}$ & 0.76 & 0.81 & $\underline{1.12}$ \\
\hline $40 \%$ & 1.44 & 1.57 & $\overline{2.30}$ & 0.87 & 0.94 & $\underline{1.35}$ & 0.72 & 0.77 & $\overline{1.10}$ & 0.64 & 0.70 & $\overline{0.98}$ \\
\hline $42 \%$ & 1.16 & 1.30 & $\underline{1.97}$ & 0.72 & 0.79 & $\underline{1.18}$ & 0.59 & 0.66 & $\underline{0.96}$ & 0.53 & 0.59 & $\underline{0.86}$ \\
\hline $44 \%$ & 0.92 & 1.07 & $\underline{1.69}$ & 0.58 & 0.66 & $\underline{1.02}$ & 0.48 & 0.55 & $\underline{0.84}$ & 0.43 & 0.49 & $\underline{0.75}$ \\
\hline $46 \%$ & 0.71 & 0.87 & $\overline{1.43}$ & 0.45 & 0.54 & $\overline{0.88}$ & 0.38 & 0.45 & $\overline{0.72}$ & 0.34 & 0.41 & $\overline{0.65}$ \\
\hline $48 \%$ & 0.53 & 0.68 & $\underline{1.21}$ & 0.34 & 0.44 & $\underline{0.75}$ & 0.29 & 0.36 & $\underline{0.62}$ & 0.26 & 0.33 & $\underline{0.56}$ \\
\hline $50 \%$ & 0.37 & 0.52 & $\overline{1.01}$ & 0.24 & 0.34 & $\overline{0.63}$ & 0.21 & 0.28 & $\overline{\underline{0.53}}$ & 0.19 & 0.26 & $\overline{0.48}$ \\
\hline
\end{tabular}


Table 12: Kappas of Average, Asian and OBPI funds in the presence of jumps: Euro Stoxx 50

\begin{tabular}{|c|c|c|c|c|c|c|c|c|c|c|c|c|}
\hline \multirow[b]{2}{*}{$L / V_{0}$} & \multicolumn{3}{|c|}{ Kappa 1} & \multicolumn{3}{|c|}{ Kappa 2} & \multicolumn{3}{|c|}{ Kappa 3} & \multicolumn{3}{|c|}{ Kappa 4} \\
\hline & Aver. & Asian & OBPI & Aver. & Asian & OBPI & Aver. & Asian & OBPI & Aver. & Asian & OBPI \\
\hline $2 \%$ & $\underline{323.3}$ & 128.4 & 155.5 & 99.25 & 60.09 & 70.65 & $\underline{65.73}$ & 46.57 & 54.20 & $\underline{53.13}$ & 40.97 & 47.45 \\
\hline $4 \%$ & $\underline{119.4}$ & 60.01 & 72.58 & $\underline{42.40}$ & 28.66 & 33.79 & $\underline{29.41}$ & 22.33 & 26.09 & $\underline{24.31}$ & 19.69 & 22.89 \\
\hline $6 \%$ & 64.48 & 37.35 & 45.19 & 25.10 & 18.21 & 21.53 & 17.93 & 14.26 & 16.72 & 15.04 & 12.60 & 14.71 \\
\hline $8 \%$ & 40.85 & 26.13 & 31.67 & $\underline{17.01}$ & 12.99 & 15.42 & $\underline{12.42}$ & 10.22 & 12.05 & 10.53 & 9.05 & $\underline{10.62}$ \\
\hline $10 \%$ & $\underline{28.23}$ & 19.48 & 23.67 & $\underline{12.41}$ & 9.87 & 11.77 & 9.23 & 7.81 & $\underline{9.25}$ & 7.89 & 6.93 & $\underline{8.17}$ \\
\hline $12 \%$ & $\underline{20.61}$ & 15.10 & 18.42 & $\underline{9.49}$ & 7.80 & 9.35 & 7.16 & 6.20 & $\underline{7.39}$ & 6.16 & 5.51 & $\underline{6.54}$ \\
\hline $14 \%$ & $\underline{15.62}$ & 12.03 & 14.74 & 7.49 & 6.33 & $\underline{7.63}$ & 5.72 & 5.06 & $\underline{6.06}$ & 4.96 & 4.51 & $\underline{5.38}$ \\
\hline $16 \%$ & $\underline{12.16}$ & 9.77 & 12.03 & 6.04 & 5.24 & $\underline{6.35}$ & 4.67 & 4.20 & $\underline{5.07}$ & 4.06 & 3.75 & $\underline{4.51}$ \\
\hline $18 \%$ & 9.65 & 8.05 & $\underline{9.97}$ & 4.95 & 4.39 & $\underline{5.36}$ & 3.86 & 3.54 & $\underline{4.30}$ & 3.38 & 3.16 & $\underline{3.83}$ \\
\hline $20 \%$ & 7.78 & 6.70 & $\underline{8.36}$ & 4.10 & 3.71 & $\underline{4.57}$ & 3.23 & 3.01 & $\underline{3.69}$ & 2.84 & 2.70 & $\underline{3.29}$ \\
\hline $22 \%$ & 6.34 & 5.62 & 7.07 & 3.43 & 3.17 & $\underline{3.93}$ & 2.73 & 2.58 & $\underline{3.19}$ & 2.41 & 2.31 & $\underline{2.85}$ \\
\hline $24 \%$ & 5.21 & 4.74 & $\underline{6.02}$ & 2.89 & 2.72 & $\underline{3.41}$ & 2.32 & 2.22 & $\underline{2.77}$ & 2.05 & 2.00 & $\underline{2.49}$ \\
\hline $26 \%$ & 4.31 & 4.02 & $\underline{5.16}$ & 2.45 & 2.34 & $\underline{2.96}$ & 1.97 & 1.92 & $\underline{2.42}$ & 1.76 & 1.73 & $\underline{2.18}$ \\
\hline $28 \%$ & 3.58 & 3.41 & $\underline{4.43}$ & 2.07 & 2.01 & $\underline{2.59}$ & 1.69 & 1.66 & $\underline{2.12}$ & 1.50 & 1.50 & $\underline{1.91}$ \\
\hline $30 \%$ & 2.97 & 2.90 & $\underline{3.82}$ & 1.76 & 1.74 & $\underline{2.27}$ & 1.44 & 1.44 & $\underline{1.87}$ & 1.29 & 1.30 & $\underline{1.68}$ \\
\hline $32 \%$ & 2.47 & 2.47 & $\underline{3.30}$ & 1.49 & 1.50 & 1.99 & 1.23 & 1.24 & $\underline{1.64}$ & 1.10 & 1.13 & $\underline{1.49}$ \\
\hline $34 \%$ & 2.05 & 2.09 & $\underline{2.86}$ & 1.26 & 1.29 & $\underline{1.74}$ & 1.04 & 1.08 & $\underline{1.45}$ & 0.94 & 0.98 & $\underline{1.31}$ \\
\hline $36 \%$ & 1.70 & 1.77 & $\underline{2.47}$ & 1.06 & 1.11 & $\underline{1.53}$ & 0.88 & 0.93 & $\underline{1.27}$ & 0.80 & 0.84 & $\underline{1.16}$ \\
\hline $38 \%$ & 1.39 & 1.49 & $\underline{2.14}$ & 0.88 & 0.94 & $\underline{1.34}$ & 0.74 & 0.79 & $\underline{1.12}$ & 0.67 & 0.72 & $\underline{1.02}$ \\
\hline $40 \%$ & 1.13 & 1.25 & $\underline{1.84}$ & 0.73 & 0.80 & $\underline{1.17}$ & 0.61 & 0.67 & $\underline{0.98}$ & 0.56 & 0.61 & $\underline{0.89}$ \\
\hline $42 \%$ & 0.91 & 1.03 & $\underline{1.58}$ & 0.59 & 0.67 & $\underline{1.02}$ & 0.50 & 0.57 & $\underline{0.86}$ & 0.46 & 0.52 & $\underline{0.78}$ \\
\hline $44 \%$ & 0.71 & 0.84 & $\underline{1.36}$ & 0.47 & 0.55 & $\underline{0.88}$ & 0.40 & 0.47 & $\underline{0.75}$ & 0.36 & 0.43 & $\underline{0.68}$ \\
\hline $46 \%$ & 0.54 & 0.67 & $\underline{1.15}$ & 0.36 & 0.45 & $\underline{0.76}$ & 0.31 & 0.38 & $\underline{0.64}$ & 0.28 & 0.35 & $\underline{0.59}$ \\
\hline $48 \%$ & 0.39 & 0.52 & $\underline{0.97}$ & 0.26 & 0.35 & $\underline{0.65}$ & 0.23 & 0.30 & $\underline{0.55}$ & 0.21 & 0.28 & $\underline{0.50}$ \\
\hline $50 \%$ & 0.26 & 0.39 & $\underline{0.81}$ & 0.18 & 0.27 & $\underline{0.55}$ & 0.15 & 0.23 & $\underline{0.47}$ & 0.14 & 0.21 & $\underline{0.43}$ \\
\hline
\end{tabular}

In Figure 4, we provide the empirical CDF of the three fund returns. We note in particular that the probability of merely recovering the guaranteed amount at maturity is always lower for the Average fund (about 0.025 for both the S\&P 500 and the Euro Stoxx 50), while, for the Asian fund, this probability is about 0.15 for the S\&P 500 and 0.13 for the Euro Stoxx 50, and for the OBPI fund, it is about 0.13 for the S\&P 500 and 0.11 for the Euro Stoxx 50. The OBPI fund provides a higher probability of obtaining high returns (for example, a probability of about 0.1 of a return higher than $50 \%$ for $T=8$ years for the S\&P 500, while this probability is about 0.09 for the Euro Stoxx 50). For the Asian and the Average funds, this probability is respectively about 0.06 and 0.05 for the S\&P 500 and about 0.05 for the Euro Stoxx 50. 


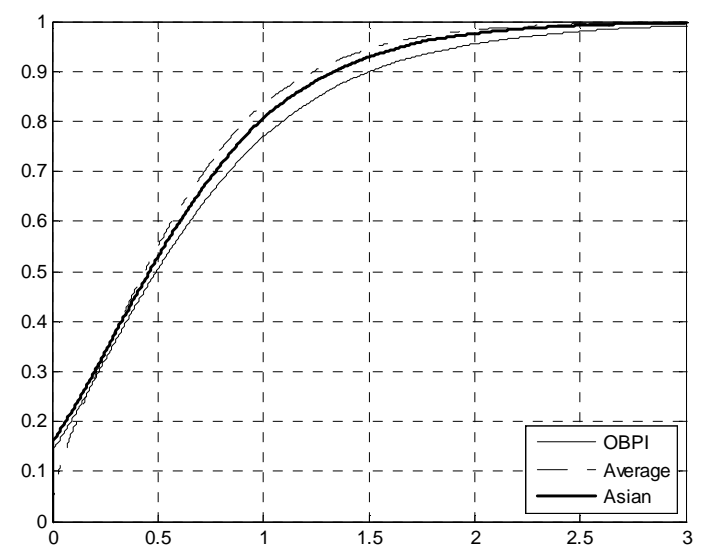

A. S\&P 500

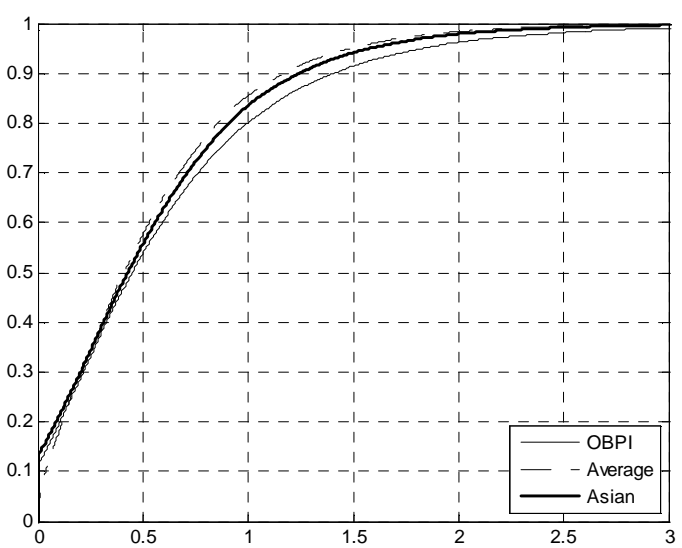

B. Euro Stoxx 50

Figure 4: Empirical CDF of the Average, the Asian and the OBPI funds

\section{Conclusion}

In this paper, we analyze two standard financial structured products both providing a guarantee for $100 \%$ of the initial investment and smoothing the fluctuations of a given underlying asset, usually a financial market index. The first fund has an optional component in the form of an Asian call option, while the second is based on an average of call options.

We show that Asian fund expected return is the highest. Thus, for investors focusing only on expected return (risk-neutral investors), Asian funds are preferred. But, as soon as potential risk aversion or implicitly loss aversion (see ranking by Kappa measures) are taken into account, the ranking is reversed. This is one of the main results of our study.

We also compare their cumulative distribution functions. We prove that the probability of providing merely the guaranteed amount at maturity is significantly higher for the Asian fund, which penalizes this latter fund. Indeed, the average of call options allow to benefit from the ratchet effect. In addition, we also analyze the standard OBPI fund whose design is much simpler. We show that its probability distribution is close to that of Asian type, especially for small return values.

We also prove that the OBPI fund can be the best fund for various Kappa measures, even though it does not provide the same hedge against the volatility of the underlying asset. Our findings show that the complexity of the Asian option (for example the dynamic hedging of such a fund) is not significantly compensated for by its actual performances. 


\section{References}

[1] Bertrand P., Prigent, J.-L. (2005). Portfolio insurance strategies: OBPI versus CPPI. Finance 26, $5-32$.

[2] Bertrand, P., Prigent, J.-L. (2011). Omega performance measure and portfolio insurance. Journal of Banking and Finance 35, 1811-1823.

[3] Bertrand, P., Prigent, J.-L. (2012). On the fair pricing of financial structured products: The French financial market case. Working paper ThEMA, University of Cergy-Pontoise.

[4] Boyle, P. P., Broadie, M., Glasserman, P. (1997). Monte Carlo methods for security pricing. Journal of Economic Dynamics and Control 21, 1267-1321.

[5] Burth, S., Kraus, T., Wohlwend, H. (2001). The pricing of structured products in the Swiss market. Journal of Derivatives 9, 30-40.

[6] Chang, E. C. ,Tang, D.Y., Zhang, M. (2013). Suitability checks and household investments in structured products. Working paper Faculty of Business and Economics. The University of Hong Kong. Forthcoming in Journal of Financial and Quantitative Finance.

[7] Chen, A. H., Kensinger, J.W. (1990). An analysis of market-index certificates of deposit. Journal of Financial Services Research 4, 93-110.

[8] Chen, K.C., Sears, R.S., (1990). Pricing the SPIN. Financial Management 19, 36-47.

[9] Das, S.R., Statman M., (2013). Options and structured products in behavioral portfolios. Journal of Economic Dynamics and Control 37, 137-153.

[10] Den Iseger, P., Oldenkamp, E. (2006). Pricing guaranteed return rate products and discretely sampled Asian options. Journal of Computational Finance 9, 1-39.

[11] Driessen, J., Maenhout, P. (2007). An empirical portfolio perspective on option pricing anomalies. Review of Finance 11, 561-603.

[12] El Karoui, N., Jeanblanc-Picqué, M., Shreve, S. (1998). Robustness of the Black and Scholes formula. Mathematical Finance 8, 93-126.

[13] Jeanblanc, M., Yor, M., Chesney, M. (2009). Mathematical Methods for Financial Markets. SpringerVerlag, London.

[14] Kaplan, P., Knowles, J. A. (2004). Kappa: A generalized downside risk-adjusted performance measure. Journal of Performance Measurement 8, 42-54.

[15] Kou, S. G., Wang, H. (2004). Option pricing under a double exponential jump diffusion model. Management Science 50, 1178-1192.

[16] Leland, H.E., Rubinstein, M. (1976). The evolution of portfolio insurance. In: D.L. Luskin, ed., Portfolio Insurance: A Guide to Dynamic Hedging, (1976), Wiley.

[17] Merton, R.C. (1976). Option pricing when underlying stock returns are discontinuous. Journal of Financial Economics 3, 125-144. 
[18] Ramezani, C., Zeng, Y. (2007). Maximum likelihood estimation of the double exponential jumpdiffusion process. Annals of Finance 3, 487-507.

[19] Shefrin, H., Statman, M. (1993). Behavioral aspects of the design and marketing of financial products. Financial Management 22, 123-134.

[20] Stoimenov, P., Wilkens, S. (2005). Are structured products 'fairly' priced? Analysis of the German market for equity-linked instruments. Journal of Banking and Finance 29, 2971-2993.

[21] Turnbull, S.T., Wakeman, L.M. (1991). A quick algorithm for pricing European average options. Journal of Financial and Quantitative Analysis 26, 377-389.

[22] Unser, M. (2000). Lower partial moments as measures of perceived risk: An experimental study. Journal of Economic Psychology 21, 253-280.

[23] Wasserfallen, W., Schenk, C. (1996). Portfolio insurance for the small investor in Switzerland. Journal of Derivatives 3, 37-43.

[24] Weber, M.,Weber, E.U., Nosic, A. (2013). Who takes risks when and why: Determinants of changes in investor risk taking. Review of Finance 17, 847-883.

[25] Zakamouline, V. (2013). Portfolio performance evaluation with loss aversion. To be published in Quantitative Finance. DOI:10.1080/14697688.2011.620978. 


\section{Appendix: Probability of obtaining merely initial value at maturity}

In this Appendix, we provide the three probabilities of merely returning initial value at maturity for the continous-time monitoring case. Even if structured products are defined from discrete-time monitoring, it is worth checking what happens if the number of dates at which intermediate performances are computed increases significantly (for example, if the frequency were weekly or even daily).

For the Asian fund, this is also a rather complex formula, while for the Average and OBPI funds, the results are quite simple to analyze. For the Average fund, the probability of obtaining merely the insured amount at maturity (here, the initial fund value ) is null.

This latter result is in line with Proposition (4) for discrete-time monitoring since it makes recourses to an Average fund more attractive. We illustrate this feature for continuous-time monitoring, assuming GBM dynamics. For all the products, we need to determine conditions corresponding to the cancellation of the optional component.

\section{Asian fund}

For the Asian fund, the probability of recovering merely the guaranteed amount at maturity is equal to:

$$
P\left[\mathcal{A}_{T}=\frac{1}{T} \int_{0}^{T} S_{s} d s \leq S_{0}\right] .
$$

Under GBM assumption, the probability distribution of $\mathcal{A}_{T}=\frac{1}{T} \int_{0}^{T} S_{s} d s$ is semi-explicit (by means of multiple integrals). From properties of the Brownian motion with drift, ${ }^{22}$ we deduce that the probability distribution of $\mathcal{A}_{T}$ is the same as the probability distribution of $\frac{4 S_{0}}{\sigma^{2} T} A_{\sigma^{2} T / 4}^{(m)}$ with $m=\frac{2 \nu}{\sigma}$ where $A_{t}^{(m)}=$ $\int_{0}^{t} \exp \left[2\left(m s+W_{s}\right)\right] d s$. Recall that the pdf $\varphi(t, u, m)$ of $A_{t}^{(m)}$ is given by:

$$
\begin{aligned}
\varphi(t, u, m)= & u^{m-1} \frac{1}{\sqrt{\left(2 \pi^{3} t\right)}} \exp \left[\frac{\pi^{2}}{2 t}-\frac{1}{2 u}-\frac{m^{2} t}{2}\right] \int_{0}^{\infty} y^{m} \exp \left[-\frac{u}{2} y^{2}\right] \Upsilon_{y}(t) d y \\
\Upsilon_{y}(t) & =\int_{0}^{\infty} \exp \left[-z^{2} / 2 t\right] \exp [-y \cosh (z)] \sinh (z) \sin (\pi z / t) d z .
\end{aligned}
$$

Therefore, we deduce that:

$$
P\left[\mathcal{A}_{T} \leq S_{0}\right]=P\left[A_{\sigma^{2} T / 4}^{(m)} \leq \sigma^{2} T / 4\right]=\int_{0}^{\sigma^{2} T / 4} \varphi\left(\sigma^{2} T / 4, u, m\right) d u,
$$

which is nonnegative.

\footnotetext{
${ }^{22}$ See Jeanblanc et al. 2009 , pp 382-385.
} 


\section{Average fund}

For the Average fund, the probability of recovering merely the guaranteed amount at maturity satisfies:

$$
P\left[\int_{0}^{T} e^{-r t} \operatorname{Max}\left(S_{t}-S_{0}, 0\right) d t=0\right]=P\left[\int_{0}^{T} \mathbb{I}_{S_{t} \leq S_{0}} d t=0\right],
$$

since both terms $e^{-r t} \operatorname{Max}\left(S_{t}-S_{0}, 0\right)$ and $\mathbb{I}_{S_{t} \leq S_{0}}$ are positive and are equal to 0 simultaneously.

Note that:

$$
P\left[\int_{0}^{T} \mathbb{I}_{S_{t} \leq S_{0}} d t=0\right] \leq P\left[\int_{0}^{T} \mathbb{I}_{S_{t}<S_{0}} d t=0\right]=P\left[\int_{0}^{T} \mathbb{I}_{\nu t+W_{t}<0} d t=0\right]
$$

Using results about occupation time for a drifted Brownian motion (see Jeanblanc et al., 2009, pp 114-115), it can be shown that $\int_{0}^{T} \mathbb{I}_{\nu t+W_{t}<0} d t$ has a density. ${ }^{23}$ This latter result proves that $P\left[\int_{0}^{T} \mathbb{I}_{\nu t+W_{t}<0} d t=0\right]=$ 0 , from which we deduce:

$$
P\left[\int_{0}^{T} e^{-r t} \operatorname{Max}\left(S_{t}-S_{0}, 0\right) d t=0\right]=0 .
$$

Thus, for the Average fund, the probability of its value winding up at the insured level is null.

\section{OBPI fund}

For the OBPI fund, the probability of recovering merely the guaranteed amount at maturity is simply equal to:

$$
P\left[S_{T} \leq S_{0}\right]=\mathcal{N}[-\nu \sqrt{T}]
$$

which is nonnegative.

This latter probability is decreasing (resp. increasing) as soon as the parameter $v=\left(\frac{\mu}{\sigma}-\frac{1}{2} \sigma\right)$ is positive (resp. negative). Condition $v>0$ corresponds to values of the drift $\mu$ that are sufficiently high compared to the volatility $\sigma$ (i.e. $\mu>\sigma^{2} / 2$ ). For example, if the volatility $\sigma$ is equal to $20 \%, v$ is positive as soon as $\mu$ is higher than $2 \%$, which is usually the case. Indeed, the higher the drift, the higher the values of the risky asset, thus the lower the probability winding up with merely the insured amount. Note also that for fixed drift and maturity, this probability is increasing with respect to the volatility.

\footnotetext{
${ }^{23}$ When the drift $\nu$ is null, the distribution of $\int_{0}^{T} \mathbb{I}_{W_{t}<0} d t$ is exactly the arcsine law on $[0, T]$ with density $\frac{d t}{\pi \sqrt{t(T-t)}} \mathbb{I}_{t<T}$.
} 